Karl-Franzens Universität Graz

Technische Universität Graz

Medizinische Universität Graz

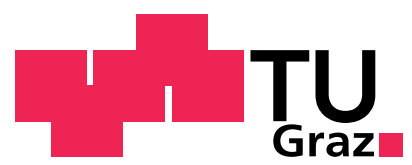

\title{
Optimal control of the bidomain system (II): Uniqueness and regularity theorems for weak solutions
}

\author{
Marcus Wagner Karl Kunisch
}


SFB sponsors:

- Austrian Science Fund (FWF)

- University of Graz

- Graz University of Technology

- Medical University of Graz

- Government of Styria

- City of Graz

Das Land

Steiermark

Stadt $\mathbf{G} \mathbf{R}$ A Z $\mathbf{Z}$ Wissenschaft 


\title{
Optimal control of the bidomain system (II):
}

\section{Uniqueness and regularity theorems for weak solutions. Revised version}

\author{
Karl Kunisch and Marcus Wagner
}

\section{Introduction.}

In the present paper, we continue our investigation of optimal control problems for the bidomain equations. In a previous work [KUNISCH/WAGNER 11], control problems involving the monodomain approximation of the bidomain system have been considered. Well-posedness of the problem formulation was proved, and first-order optimality conditions were derived. Turning now to the study of optimal control of the full bidomain system, the present work is focussed on the uniqueness, stability and regularity analysis for weak solutions of the system, thus establishing the well-posedness of the control-to-state mapping and examinating the possible gain of regularity for solutions, which satisfy the optimality conditions. At the same time, the stability estimate proved below forms itself the first part of the proof of the necessary optimality conditions for the problem $(\mathrm{P})$ below. The investigation of the control problem will be continued in a subsequent publication with proving existence of global minimizers and completing the proof of the first-order optimality conditions. For a bounded domain $\Omega \subset \mathbb{R}^{3}$ and $T>0$, the bidomain system reads as follows: ${ }^{01 \text { ) }}$

$$
\begin{aligned}
& (\mathrm{B})_{0} \frac{\partial \Phi_{t r}}{\partial t}+I_{i o n}\left(\Phi_{t r}, W\right)-\operatorname{div}\left(M_{i} \nabla \Phi_{i}\right)=\quad I_{i} \quad \text { for almost all }(x, t) \in \Omega \times[0, T] \text {; } \\
& \frac{\partial \Phi_{t r}}{\partial t}+I_{i o n}\left(\Phi_{t r}, W\right)+\operatorname{div}\left(M_{e} \nabla \Phi_{e}\right)=-I_{e} \quad \text { for almost all }(x, t) \in \Omega \times[0, T] \\
& \frac{\partial W}{\partial t}+G\left(\Phi_{t r}, W\right) \quad=\quad 0 \text { for almost all }(x, t) \in \Omega \times[0, T] ; \\
& \mathfrak{n}^{\mathrm{T}} M_{i} \nabla \Phi_{i}=0 \text { for all }(x, t) \in \partial \Omega \times[0, T] \\
& \mathfrak{n}^{\mathrm{T}} M_{e} \nabla \Phi_{e}=0 \quad \text { for all }(x, t) \in \partial \Omega \times[0, T] \\
& \Phi_{t r}(x, 0)=\Phi_{i}(x, 0)-\Phi_{e}(x, 0)=\Phi_{0}(x) \quad \text { and } \quad W(x, 0)=W_{0}(x) \quad \text { for almost all } x \in \Omega .
\end{aligned}
$$

Here $\Omega$ represents the domain occupied by the cardiac muscle, the variables $\Phi_{i}$ and $\Phi_{e}$ denote the intracellular and extracellular electric potentials, and $\Phi_{t r}=\Phi_{i}-\Phi_{e}$ is the transmembrane potential. The ionic current $I_{i o n}$ and the function $G$ within the gating equation (1.3) will be specified according to the two-variable models discussed in Subsection 2.b) below. In these models, $I_{i o n}$ and $G$ depend on $\Phi_{t r}$ as well as on a single gating variable $W$ describing in a cumulative way the effects of the ion transport through the cell membranes. The anisotropic properties of the intracellular and the extracellular tissue parts are modeled by the conductivity tensors $M_{i}$ and $M_{e}$. Finally, $I_{i}$ and $I_{e}$ describe the intracellular and extracellular stimulation currents, respectively.

01) First considered in [Tung 78] . A detailed introduction may be found in [SUNDnEs/Lines/CAI/NiELSEN/MARDAL/ Tveito 06], pp. $21-56$. 
As already mentioned, our aim is to analyze optimal control problems related to (1.1) - (1.6):

(P) $F\left(\Phi_{t r}, \Phi_{e}, W, I_{e}\right)=\int_{0}^{T} \int_{\Omega} r\left(x, t, \Phi_{t r}(x, t), \Phi_{e}(x, t), W(x, t)\right) d x d t+\frac{\mu}{2} \int_{0}^{T} \int_{\Omega} I_{e}(x, t)^{2} d x d t \longrightarrow \inf$ ! subject to the bidomain equations $(1.1)-(1.6)$ in its weak formulation (see $(2.7)-(2.10)$ below)

and the control restriction $\left|I_{e}(x, t)\right| \leqslant R$ for almost all $(x, t) \in \Omega \times[0, T]$.

In this problem, the bidomain system will be controlled by means of the extracellular excitation $I_{e}{ }^{02)}$ Within the objective (1.7), the first term with the integrand $r(x, t, \varphi, \eta, w)$ can be considered as a data term, e. g. of tracking type. The inclusion of the control into the cost functional (1.7) and the pointwise control restriction (1.8) serve different modeling purposes. While the former models the requirement that regardless of whether the hard bound (1.8) is active - the overall stimulus should be as small as possible, thus favoring solutions with little intervention to the cardiac system, the hard constraint (1.8) for the extracellular stimulus reflects the obvious fact that electrical stimulations applied to living tissue cannot be arbitrarily large without causing damage. In mathematical terms, either the control term within (1.7) or the pointwise constraint (1.8) is enough to ensure the existence of a weakly convergent minimizing sequence.

In the framework of optimal control, it turns out that the appropriate solution concept for the bidomain system is the concept of weak solutions. This is sufficient to establish existence results for $(\mathrm{P})$, $\left.{ }^{03}\right) \mathrm{A}$ first natural question is then whether the control-to-state mapping, which assigns to a given control $I_{e}$ the state $\left(\Phi_{t r}, \Phi_{e}, W\right)$, is well-posed; in other words, whether existence and uniqueness of weak solutions of the bidomain equations in correspondence to right-hand sides $I_{i}=\mathfrak{o}$ and $I_{e}$ can be guaranteed. Let us briefly discuss the related results available in the literature. In [BOURGAULT/COUdièrE/PIERRE 09], global existence of weak solutions for the two-variable models from Subsection 2.b) was obtained. This result is cited below as Theorem 2.5. A sufficient condition for uniqueness based on eigenvalue stability of a matrix related to $\left(\begin{array}{c}c \partial I_{i o n} / \partial \varphi c \partial I_{i o n} / \partial w \\ \partial G / \partial \varphi \\ \partial G / \partial w\end{array}\right)$, however, could be proven only for the FitzHugh-Nagumo model. ${ }^{04)}$ Similar results can be found in [Boulakia/Fernández/GERBEAU/ZEMZEmi 08], namely a global existence theorem for weak solutions together with a uniqueness result requiring spectral conditions analogously to the former ones. ${ }^{05)}$ For the FitzHugh-Nagumo model, the results and methods from [Bourgault/Coudière/PIERRE 09 ] have been predated in [COLLI FRAnZONE/SAVARÉ 02] where existence and uniqueness of weak solutions has been established. ${ }^{06)}$ Further, in [Bourgault/Coudière/Pierre 09], existence and uniqueness of strong solutions is verified locally in time. ${ }^{07)}$ Global existence and uniqueness of strong solutions has been proved in [VENERONi 09] for a generalized Luo-Rudy type model. ${ }^{08)}$

Our second aim, to be addressed in an upcoming paper, is to provide a rigorous proof of necessary optimality conditions for the control problem (P). As suggested by [KUNISCH/WAGNER 11], the problem can be treated then as a so-called weakly singular problem. Within this approach, a stability estimate for the bidomain

02) For physiological reasons, the intracellular excitation $I_{i}$ must be set zero.

03) Cf. [Kunisch/Wagner 11], p. 8, Theorem 3.3.

04) [Bourgault/Coudière/Pierre 09], p. 479, Theorem 32, together with Subsect. 6.1., p. 479 f.

05) [Boulakia/Fernández/Gerbeau/Zemzemi 08] , p. 9, Theorem 2.2. Besides the models from Subsection 2.b), the authors consider the Mitchell-Schaeffer model.

06) [Colli Franzone/Savaré 02], p. 64, Theorem 2.

07) [Bourgault/Coudière/Pierre 09] , p. 469, Theorem 20. The assumptions of this theorem are rather difficult to verify.

08) [Veneroni 09], p. 854, Theorem 1.1. 
equations is required. ${ }^{09)}$ Moreover, we will investigate regularity properties of an optimal control resulting from the first-order necessary optimality conditions. ${ }^{10)}$

Consequently, the present work is concerned with the proof of a global existence and uniqueness theorem for weak solutions for two-variable models, as discussed e. g. in [Bourgault/Coudière/Pierre 09] (Theorem 2.8.). The assumptions for this theorem are the natural ones for the context of optimal control. Namely, in view of (1.8), we assume that the inhomogeneities $I_{i}$ and $I_{e}$ belong to $L^{\infty}\left[(0, T),\left(W^{1,2}(\Omega)\right)^{*}\right]$. Instead of spectral conditions of the type discussed above, our uniqueness argument is based on the proof of a stability estimate (Theorem 2.7.). At the same time, this theorem forms the first part of the proof of the necessary optimality conditions for the control problem (P), which will be completed in an upcoming publication. Further, in the case of controls admitting a weak time derivative, we will prove that the weak solutions of the bidomain equations gain additional regularity as well and become, in fact, strong solutions of (1.1) - (1.6) (Theorem 3.3. and Corollary 3.4.). The proof is based on an adaptation of the iterative procedure presented in [Veneroni 09] .

The paper is structured as follows: In Section 2, we introduce the strong and weak solution concepts and present the models for the ionic current, namely the Rogers-McCulloch, FitzHugh-Nagumo and the linearized Aliev-Panfilov model. Then we proceed with the existence and uniqueness analysis of weak solutions. Higher regularity of these solutions is investigated in Section 3. The assumptions and results are presented at the beginning of each sections, while the rather technical proofs are given at their ends. All proofs will be completely worked out for the Rogers-McCulloch model. For the FitzHugh-Nagumo and the linearized Aliev-Panfilov model, the necessary alterations will be indicated.

\section{Notations.}

We denote by $L^{p}(\Omega)$ the space of functions, which are in the $p$ th power integrable $(1 \leqslant p<\infty)$, or are measurable and essentially bounded $(p=\infty)$, and by $W^{1, p}(\Omega)$ the Sobolev space of functions $\psi: \Omega \rightarrow \mathbb{R}$ which, together with their first-order weak partial derivatives, belong to the space $L^{p}(\Omega, \mathbb{R})(1 \leqslant p<$ $\infty)$. For spaces of Bochner integrable mappings, e. g. $L^{2}\left[(0, T), W^{1,2}(\Omega)\right]$, we refer to the summary in [KUnisCh/WAGNeR 11], p. $20 \mathrm{f} . \Omega_{T}$ is an abbreviation for $\Omega \times[0, T]$. The gradient $\nabla$ is always taken only with respect to the spatial variables $x$. The nonstandard abbreviation " $(\forall) t \in \mathrm{A}$ " has to be read as "for almost all $t \in \mathrm{A}$ " or "for all $t \in \mathrm{A}$ except for a Lebesgue null set", and the symbol $\mathfrak{o}$ denotes, depending on the context, the zero element or the zero function of the underlying space.

\section{Existence and uniqueness of weak solutions for the bidomain system.}

\section{a) Equivalent formulations of the bidomain system; strong and weak solutions.}

Introducing the transmembrane potential $\Phi_{t r}=\Phi_{i}-\Phi_{e}$, the bidomain system (1.1) - (1.6) can be equivalently stated in the following parabolic-elliptic form: ${ }^{11)}$

09) Analogously to [Kunisch/Wagner 11], p. 11, Theorem 3.8., and p. 16, Proof of Theorem 3.5., Step 2. For the general framework of weakly singular problems, cf. [ITO/KunisCH 08], p. $17 \mathrm{f}$.

10) In the case of the monodomain approximation, a situation with improved regularity of the optimal control has been described in [Kunisch/WAGNER 11], p. 10, Corollary 3.7.

11) See [Bourgault/Coudière/Pierre 09] , p. 459. 
(B) $)_{1} \frac{\partial \Phi_{t r}}{\partial t}+I_{i o n}\left(\Phi_{t r}, W\right)-\operatorname{div}\left(M_{i} \nabla \Phi_{t r}\right)-\operatorname{div}\left(M_{i} \nabla \Phi_{e}\right)=I_{i}$ for almost all $(x, t) \in \Omega \times[0, T] ;$

$\operatorname{div}\left(M_{i} \nabla \Phi_{t r}\right)+\operatorname{div}\left(\left(M_{i}+M_{e}\right) \nabla \Phi_{e}\right)=-\left(I_{i}+I_{e}\right) \quad$ for almost all $(x, t) \in \Omega \times[0, T] ;$

$\frac{\partial W}{\partial t}+G\left(\Phi_{t r}, W\right)=0$ for almost all $(x, t) \in \Omega \times[0, T]$

$\mathfrak{n}^{\mathrm{T}} M_{i} \nabla \Phi_{t r}+\mathfrak{n}^{\mathrm{T}} M_{i} \nabla \Phi_{e}=0 \quad$ for all $(x, t) \in \partial \Omega \times[0, T]$

$\mathfrak{n}^{\mathrm{T}} M_{i} \nabla \Phi_{t r}+\mathfrak{n}^{\mathrm{T}}\left(M_{i}+M_{e}\right) \nabla \Phi_{e}=0 \quad$ for all $(x, t) \in \partial \Omega \times[0, T] ;$

$\Phi_{t r}(x, 0)=\Phi_{0}(x) \quad$ and $\quad W(x, 0)=W_{0}(x) \quad$ for almost all $x \in \Omega$.

To the system (2.1) - (2.6), the following weak formulation corresponds: ${ }^{12)}$

$(\mathrm{B})_{2} \quad \int_{\Omega}\left(\frac{\partial \Phi_{t r}}{\partial t} \cdot \psi+\nabla \psi^{\mathrm{T}} M_{i}\left(\nabla \Phi_{t r}+\nabla \Phi_{e}\right)+I_{i o n}\left(\Phi_{t r}, W\right) \psi\right) d x=\int_{\Omega} I_{i} \psi d x$ $\forall \psi \in W^{1,2}(\Omega)$, for a. a. $t \in(0, T) ;$

$\int_{\Omega}\left(\nabla \psi^{\mathrm{T}} M_{i} \nabla \Phi_{t r}+\nabla \psi^{\mathrm{T}}\left(M_{i}+M_{e}\right) \nabla \Phi_{e}\right) d x=\int_{\Omega}\left(I_{i}+I_{e}\right) \psi d x$ $\forall \psi \in W^{1,2}(\Omega)$ with $\int_{\Omega} \psi(x) d x=0$, for a. a. $t \in(0, T) ;$

$\int_{\Omega}\left(\frac{\partial W}{\partial t}+G\left(\Phi_{t r}, W\right)\right) \psi d x=0 \quad \forall \psi \in L^{2}(\Omega)$, for a. a. $t \in(0, T)$

$\Phi_{t r}(x, 0)=\Phi_{0}(x)$ and $W(x, 0)=W_{0}(x) \quad$ for almost all $x \in \Omega$.

The corresponding notions of strong and weak solutions will be clarified by the following definitions.

Definition 2.1. (Strong solution of the bidomain system $\left.(\mathrm{B})_{1}\right)^{13)}$ A triple $\left(\Phi_{t r}, \Phi_{e}, W\right)$ is called a strong solution of the bidomain system $(2.1)-(2.6)$ on $[0, T]$ iff the functions $\Phi_{t r}, \Phi_{e}$ and $W$ satisfy the equations $(2.1)-(2.3)$ a. e. on $\Omega \times[0, T]$ as well as the initial and boundary conditions (2.4) - (2.6) on $\partial \Omega \times[0, T]$, respectively. Moreover, the functions belong to the spaces

$$
\begin{aligned}
& \Phi_{t r} \in L^{2}\left[(0, T), W^{2,2}(\Omega)\right] \cap W^{1,2}\left[(0, T), L^{2}(\Omega)\right] ; \\
& \Phi_{e} \in L^{2}\left[(0, T), W^{2,2}(\Omega)\right] ; \\
& W \in W^{1,2}\left[(0, T), L^{2}(\Omega)\right],
\end{aligned}
$$

and for all $t \in(0, T)$, it holds that $\int_{\Omega} \Phi_{e}(x, t) d x=0$.

Note that the imbedding $W^{1,2}\left[(0, T), L^{2}(\Omega)\right] \hookrightarrow C^{0}\left[[0, T], L^{2}(\Omega)\right]$ holds; ${ }^{14)}$ consequently, the initial conditions for $\Phi_{t r}$ and $W$ are well-posed.

Definition 2.2. (Weak solution of the bidomain system $\left.(\mathrm{B})_{2}\right)^{15)}$ A triple $\left(\Phi_{t r}, \Phi_{e}, W\right)$ is called a weak solution of the bidomain system $(2.7)-(2.10)$ on $[0, T]$ iff the functions $\Phi_{t r}, \Phi_{e}$ and $W$ satisfy equations (2.7) - (2.9) and obey the initial conditions (2.10). Moreover, the functions belong to the spaces

12) Following [Bourgault/Coudière/Pierre 09], p. 472, Remark 27, the occuring distributional time derivatives can be identified as functions $\partial \Phi_{t r} / \partial t, \partial W / \partial t \in L^{2}\left[(0, T),\left(W^{1,2}(\Omega)\right)^{*}\right]$.

13) Slightly modified from [Bourgault/Coudière/Pierre 09], p. 469, Definition 18.

14) [Kunisch/Wagner 11] , p. 21, Theorem 4.5.

15) [Bourgault/Coudière/Pierre 09] , p. 472, Definition 26. 


$$
\begin{aligned}
& \Phi_{t r} \in C^{0}\left[[0, T], L^{2}(\Omega)\right] \cap L^{2}\left[(0, T), W^{1,2}(\Omega)\right] \cap L^{p}\left(\Omega_{T}\right) \text { with } 2 \leqslant p \leqslant 6 \\
& \Phi_{e} \in L^{2}\left[(0, T), W^{1,2}(\Omega)\right] \\
& W \in C^{0}\left[[0, T], L^{2}(\Omega)\right]
\end{aligned}
$$

and for almost all $t \in(0, T)$, it holds that $\int_{\Omega} \Phi_{e}(x, t) d x=0$.

Assumptions 2.3. (Basic assumptions on the data in $(\mathrm{B})_{1}$ and $(\mathrm{B})_{2}$ )

1) $\Omega \subset \mathbb{R}^{3}$ is a bounded Lipschitz domain.

2) $M_{i}, M_{e}: \operatorname{cl}(\Omega) \rightarrow \mathbb{R}^{3 \times 3}$ are symmetric, positive definite matrix functions with $L^{\infty}(\Omega)$-coefficients, obeying the uniform ellipticity conditions

$0 \leqslant \mu_{1}\|\xi\|^{2} \leqslant \xi^{\mathrm{T}} M_{i}(x) \xi \leqslant \mu_{2}\|\xi\|^{2}$ and $0 \leqslant \mu_{1}\|\xi\|^{2} \leqslant \xi^{\mathrm{T}} M_{e}(x) \xi \leqslant \mu_{2}\|\xi\|^{2} \quad \forall \xi \in \mathbb{R}^{3} \quad \forall x \in \Omega$

with $\mu_{1}, \mu_{2}>0$.

3) The initial values $\Phi_{0}, W_{0}$ belong to the space $L^{2}(\Omega)$.

4) $I_{i}$ and $I_{e}$ belong to the space $L^{2}\left[(0, T),\left(W^{1,2}(\Omega)\right)^{*}\right]$ and satisfy the compatibility condition

$$
\int_{\Omega}\left(I_{i}(x, t)+I_{e}(x, t)\right) d x=0 \quad \text { for almost all } t \in(0, T) .
$$

Under these assumptions, the weak bidomain system $(2.7)-(2.10)$ allows for a further reformulation, which is crucial for establishing existence and uniqueness results.

Theorem 2.4. (The reduced bidomain system and the bidomain bilinear form) 1) ${ }^{16)}$ Under the Assumptions 2.3., a triple $\left(\Phi_{t r}, \Phi_{e}, W\right)$ forms a weak solution of $(\mathrm{B})_{2}$ on the interval $[0, T]$ for the initial values $\Phi_{0}, W_{0} \in L^{2}(\Omega)$ iff it satisfies the reduced bidomain system

$$
\begin{gathered}
(\mathrm{B})_{3} \quad \frac{d}{d t}\left\langle\Phi_{t r}(t), \psi\right\rangle+A\left(\Phi_{t r}(t), \psi\right)+\int_{\Omega} I_{i o n}\left(\Phi_{t r}(t), W(t)\right) \psi d x=\langle S(t), \psi\rangle \quad \forall \psi \in W^{1,2}(\Omega) ; \\
\frac{d}{d t}\langle W(t), \psi\rangle+\int_{\Omega} G\left(\Phi_{t r}(t), W(t)\right) \psi d x=0 \quad \forall \psi \in L^{2}(\Omega) \\
\Phi_{t r}(x, 0)=\Phi_{0}(x) \quad \text { and } \quad W(x, 0)=W_{0}(x) \quad(\forall) x \in \Omega
\end{gathered}
$$

on $[0, T]$ in distributional sense. Here the bidomain bilinear form $A: W^{1,2}(\Omega) \times W^{1,2}(\Omega) \rightarrow \mathbb{R}$ is defined through

$$
A\left(\psi_{1}, \psi_{2}\right)=\int_{\Omega} \nabla \psi_{1}^{\mathrm{T}} M_{i} \nabla \psi_{2} d x+\int_{\Omega} \nabla \widetilde{\psi}_{e}^{\mathrm{T}} M_{i} \nabla \psi_{2} d x
$$

where $\widetilde{\psi}_{e} \in W^{1,2}(\Omega)$ is the uniquely determined solution of the variational equation

$$
\int_{\Omega} \nabla \widetilde{\psi}_{e}^{\mathrm{T}}\left(M_{i}+M_{e}\right) \nabla \psi d x=-\int_{\Omega} \nabla \psi_{1}^{\mathrm{T}} M_{i} \nabla \psi d x \quad \forall \psi \in W^{1,2}(\Omega) .
$$

Accordingly, the linear functionals $S(t) \in\left(W^{1,2}(\Omega)\right)^{*}$ are defined through

$$
\langle S(t), \psi\rangle=\left\langle I_{i}(t), \psi\right\rangle-\int_{\Omega} \nabla \bar{\psi}_{e}^{\mathrm{T}} M_{i} \nabla \psi d x
$$

16) $\overline{\text { [Bourgault/Coudière/Pierre 09] }}$, p. 473, Lemma 28, together with p. 464, Definition 5. 
where $\bar{\psi}_{e} \in W^{1,2}(\Omega)$ is the uniquely determined solution of the variational equation

$$
\int_{\Omega} \nabla \bar{\psi}_{e}^{\mathrm{T}}\left(M_{i}+M_{e}\right) \nabla \psi d x=\left\langle I_{i}(t)+I_{e}(t), \psi\right\rangle \forall \psi \in W^{1,2}(\Omega) \text { with } \int_{\Omega} \bar{\psi}_{e}(x, t) d x=0(\forall) t \in(0, T) .
$$

2) ${ }^{17)}$ The bilinear form $A$ is symmetric, continuous and coercive with

$$
\begin{aligned}
& \beta\|\psi\|_{W^{1,2}(\Omega)}^{2} \leqslant A(\psi, \psi)+\beta\|\psi\|_{L^{2}(\Omega)}^{2} \quad \forall \psi \in W^{1,2}(\Omega) \quad \text { and } \\
& \left|A\left(\psi_{1}, \psi_{2}\right)\right| \leqslant \gamma \cdot\left\|\psi_{1}\right\|_{W^{1,2}(\Omega)} \cdot\left\|\psi_{2}\right\|_{W^{1,2}(\Omega)} \quad \forall \psi_{1}, \psi_{2} \in W^{1,2}(\Omega)
\end{aligned}
$$

where $\beta, \gamma>0$.

\section{b) The models for the ionic current.}

For the ionic current $I_{i o n}$ and the function $G$ within the gating equation, we will consider the following three models:

a) The Rogers-McCulloch model. ${ }^{18)}$

$$
\begin{aligned}
& I_{i o n}(\varphi, w)=b \cdot \varphi(\varphi-a)(\varphi-1)+\varphi \cdot w=b \varphi^{3}-(a+1) b \varphi^{2}+a b \varphi+\varphi w \\
& G(\varphi, w)=\varepsilon w-\varepsilon \kappa \varphi
\end{aligned}
$$

with $0<a<1, b>0, \kappa>0$ and $\varepsilon>0$. Consequently, the gating variable obeys the linear ODE

$$
\partial W / \partial t+\varepsilon W=\varepsilon \kappa \Phi_{t r} .
$$

b) The FitzHugh-Nagumo model. ${ }^{19)}$

$$
\begin{aligned}
& I_{i o n}(\varphi, w)=\varphi(\varphi-a)(\varphi-1)+w=\varphi^{3}-(a+1) \varphi^{2}+a \varphi+w \\
& G(\varphi, w)=\varepsilon w-\varepsilon \kappa \varphi
\end{aligned}
$$

with $0<a<1, \kappa>0$ and $\varepsilon>0$. Consequently, the gating variable obeys the same linear ODE (2.30) as before.

c) The linearized Aliev-Panfilov model. ${ }^{20)}$

$$
\begin{aligned}
& I_{i o n}(\varphi, w)=b \cdot \varphi(\varphi-a)(\varphi-1)+\varphi \cdot w=b \varphi^{3}-(a+1) b \varphi^{2}+a b \varphi+\varphi w \\
& G(\varphi, w)=\varepsilon w-\varepsilon \kappa\left((a+1) \varphi-\varphi^{2}\right)
\end{aligned}
$$

with $0<a<1, b>0, \kappa>0$ and $\varepsilon>0$. The linear ODE for the gating variable is

$$
\partial W / \partial t+\varepsilon W=\varepsilon \kappa\left((a+1) \Phi_{t r}-\Phi_{t r}^{2}\right) .
$$

17) [Bourgault/Coudière/Pierre 09] , p. 464, Theorem 6.

18) [Rogers/MCCulloch 94].

19) [FitzHugh 61], together with [Nagumo/Arimoto/Yoshizawa 62].

20) See [Aliev/Panfilov 96]. The linearized model is taken from [Bourgault/Coudière/Pierre 09], p. 480. Instead, the original model contains a Riccati equation for the gating variable. 


\section{c) Existence of weak solutions.}

The following Theorems 2.5. and 2.6. were obtained in [Bourgault/CoudiÈre/PierRe 09] and [NAGaiah/Kunisch/Plank 11] .

Theorem 2.5. (Existence of weak solutions) ${ }^{21)}$ Assume that the data within $(\mathrm{B})_{2}$ obey Assumptions 2.3., and specify any of the three models described in Subsection 2.b). Then for arbitrary initial values $\Phi_{0}$, $W_{0} \in L^{2}(\Omega)$, the bidomain system $(\mathrm{B})_{2}$ admits on $[0, T]$ at least one weak solution $\left(\Phi_{t r}, \Phi_{e}, W\right)$ according to Definition 2.2. where $p=4$.

Theorem 2.6. (A priori estimate for weak solutions) ${ }^{22)}$ Assume that the data within $(\mathrm{B})_{2}$ obey Assumptions 2.3., and specify any of the three models described in Subsection 2.b). If a triple

$$
\begin{aligned}
\left(\Phi_{t r}, \Phi_{e}, W\right) \in\left(C^{0}\left[[0, T), L^{2}(\Omega)\right] \cap L^{2}[\right. & \left.\left.(0, T), W^{1,2}(\Omega)\right] \cap L^{p}\left(\Omega_{T}\right)\right) \\
& \times L^{2}\left[(0, T), W^{1,2}(\Omega)\right] \times C^{0}\left[[0, T], L^{2}(\Omega)\right]
\end{aligned}
$$

forms a weak solution of the bidomain system $(\mathrm{B})_{2}$ on $[0, T]$ then the following estimate holds:

$$
\begin{aligned}
& \left\|\Phi_{t r}\right\|_{C^{0}\left[[0, T), L^{2}(\Omega)\right]}^{2}+\left\|\Phi_{t r}\right\|_{L^{2}\left[(0, T), W^{1,2}(\Omega)\right]}^{2}+\left\|\Phi_{t r}\right\|_{L^{p}\left(\Omega_{T}\right)}^{p}+\left\|\partial \Phi_{t r} / \partial t\right\|_{L^{q}\left[(0, T),\left(W^{1,2}(\Omega)\right)^{*}\right]}^{q}{ }^{*}{ }^{2} \\
& \quad+\left\|\Phi_{e}\right\|_{L^{2}\left[(0, T), W^{1,2}(\Omega)\right]}^{2}+\|W\|_{C^{0}\left[[0, T), L^{2}(\Omega)\right]}^{+}+\|\partial W / \partial t\|_{L^{2}\left[(0, T),\left(W^{1,2}(\Omega)\right)^{*}\right]}^{2} \\
& \leqslant C \cdot\left(1+\left\|\Phi_{0}\right\|_{L^{2}(\Omega)}^{2}+\left\|W_{0}\right\|_{L^{2}(\Omega)}^{2}+\left\|I_{i}\right\|_{L^{2}\left[(0, T),\left(W^{1,2}(\Omega)\right)^{*}\right]}^{2}+\left\|I_{e}\right\|_{L^{2}\left[(0, T),\left(W^{1,2}(\Omega)\right)^{*}\right]}^{2}\right)
\end{aligned}
$$

where $p=4$ and $q=4 / 3$. The constant $C>0$ does not depend on $\Phi_{0}, W_{0}, I_{i}$ and $I_{e}$.

\section{d) Uniqueness of weak solutions.}

Note that Theorem 2.5. does not guarantee the uniqueness of the weak solution of $(2.7)-(2.10)$ in correspondence to a given pair of excitation variables $I_{i}, I_{e}$. The following error estimate, using assumptions, which are in accordance with the analytical framework of the optimal control problem $(\mathrm{P})$, allows to prove a unique correspondence between $\left(I_{i}, I_{e}\right)$ and the weak solution $\left(\Phi_{t r}, \Phi_{e}, W\right)$.

Theorem 2.7. (Stability estimate for weak solutions) Assume that the data within (B) $)_{2}$ obey Assumptions 2.3., and specify within $(\mathrm{B})_{2}$ any of the three models from Subsection 2.b). If two weak solutions $\left(\Phi_{t r}{ }^{\prime}, \Phi_{e}{ }^{\prime}, W^{\prime}\right),\left(\Phi_{t r}{ }^{\prime \prime}, \Phi_{e}{ }^{\prime \prime}, W^{\prime \prime}\right)$ of $(\mathrm{B})_{2}$ correspond to initial values $\Phi_{0}^{\prime}=\Phi_{0}^{\prime \prime}=\Phi_{0} \in L^{2}(\Omega)$, $W_{0}^{\prime}=W_{0}^{\prime \prime}=W_{0} \in L^{4}(\Omega)$ and inhomogeneities $I_{i}{ }^{\prime}, I_{e}{ }^{\prime}, I_{i}{ }^{\prime \prime}$ and $I_{e}{ }^{\prime \prime} \in L^{\infty}\left[(0, T),\left(W^{1,2}(\Omega)\right)^{*}\right]$, whose norms are bounded by $R>0$, then the following estimates hold:

$$
\begin{aligned}
& \left.\left\|\Phi_{t r}{ }^{\prime}-\Phi_{t r}{ }^{\prime \prime}\right\|_{L^{2}\left[(0, T), W^{1,2}(\Omega)\right.}^{2}+\left\|\Phi_{t r}{ }^{\prime}-\Phi_{t r}{ }^{\prime \prime}\right\|_{C^{0}\left[[0, T], L^{2}(\Omega)\right.}^{2}+\left\|\Phi_{e}{ }^{\prime}-\Phi_{e}{ }^{\prime \prime}\right\|_{L^{2}\left[(0, T), W^{1,2}(\Omega)\right.}^{2}\right] \\
& \left.+\left\|W^{\prime}-W^{\prime \prime}\right\|_{L^{2}\left[(0, T), L^{2}(\Omega)\right.}^{2}+\left\|W^{\prime}-W^{\prime \prime}\right\|_{C^{0}\left[[0, T], L^{2}(\Omega)\right.}^{2}+\left\|W^{\prime}-W^{\prime \prime}\right\|_{W^{1,2}\left[(0, T), L^{2}(\Omega)\right.}^{2}\right] \\
& \leqslant C\left(\left\|I_{i}{ }^{\prime}-I_{i}^{\prime \prime}\right\|_{L^{\infty}\left[(0, T),\left(W^{1,2}(\Omega)\right)^{*}\right]}^{2}+\left\|I_{e}{ }^{\prime}-I_{e}{ }^{\prime \prime}\right\|_{L^{\infty}\left[(0, T),\left(W^{1,2}(\Omega)\right)^{*}\right]}^{2}\right) . \\
& \left\|\Phi_{t r}{ }^{\prime}-\Phi_{t r}{ }^{\prime \prime}\right\|_{W^{1,4 / 3}\left[(0, T),\left(W^{1,2}(\Omega)\right)^{*}\right]} \leqslant C \cdot \operatorname{Max}\left(\left\|I_{i}{ }^{\prime}-I_{i}{ }^{\prime \prime}\right\|_{L^{2}\left[(0, T),\left(W^{1,2}(\Omega)\right)^{*}\right]},\right. \\
& \left.\left\|I_{e}{ }^{\prime}-I_{e}{ }^{\prime \prime}\right\|_{L^{2}\left[(0, T),\left(W^{1,2}(\Omega)\right)^{*}\right]},\left\|I_{i}{ }^{\prime}-I_{i}{ }^{\prime \prime}\right\|_{L^{2}\left[(0, T),\left(W^{1,2}(\Omega)\right)^{*}\right]}^{2},\left\|I_{e}{ }^{\prime}-I_{e}{ }^{\prime \prime}\right\|_{L^{2}\left[(0, T),\left(W^{1,2}(\Omega)\right)^{*}\right]}^{2}\right) \text {. }
\end{aligned}
$$

21) [Bourgault/Coudière/Pierre 09] , p. 473, Theorem 30.

22) [Nagaiah/Kunisch/Plank 11], p. 158, Lemma 3.5. 
The constant $C>0$ does not depend on $I_{i}{ }^{\prime}, I_{e}{ }^{\prime}, I_{i}{ }^{\prime \prime}$ and $I_{e}{ }^{\prime \prime}$ but possibly on $\Omega, R, \Phi_{0}, W_{0}$ and $p=4$.

This estimate yields the following uniqueness theorem:

Theorem 2.8. (Uniqueness of weak solutions) Assume that the data within $(\mathrm{B})_{2}$ obey Assumptions 2.3., and specify within (B) $)_{2}$ any of the three models from Subsection 2.b). Then for initial values $\Phi_{0} \in L^{2}(\Omega)$, $W_{0} \in L^{4}(\Omega)$ and inhomogeneities $I_{i}, I_{e} \in L^{\infty}\left[(0, T),\left(W^{1,2}(\Omega)\right)^{*}\right]$, the bidomain system $(\mathrm{B})_{2}$ admits on $[0, T]$ a unique weak solution $\left(\Phi_{t r}, \Phi_{e}, W\right)$ according to Definition 2.2. where $p=4$.

\section{e) Proofs.}

Throughout the following proofs, $C$ denotes a generical positive constant, which may appropriately change from line to line. $C$ will never depend on the data $I_{i}{ }^{\prime}, I_{e}{ }^{\prime}, I_{i}{ }^{\prime \prime}$ and $I_{e}{ }^{\prime \prime}$ but possibly on $\Omega, R, \Phi_{0}, W_{0}$ and $p=4$. We start with two lemmas, which will be used within the proof of Theorem 2.7.

Lemma 2.9. Under the assumptions of Theorem 2.7., let the pairs $\left(\Phi_{t r}{ }^{\prime}, W^{\prime}\right)$ and $\left(\Phi_{t r}{ }^{\prime \prime}, W^{\prime \prime}\right)$ satisfy for almost all $t \in[0, T]$ the equations

$\left\langle\frac{d}{d t} \Phi_{t r}{ }^{\prime}(t), \psi\right\rangle+A\left(\Phi_{t r}{ }^{\prime}(t), \psi\right)+\int_{\Omega} I_{i o n}\left(\Phi_{t r}{ }^{\prime}(t), W^{\prime}(t)\right) \psi d x=\left\langle S^{\prime}(t), \psi\right\rangle \quad \forall \psi \in W^{1,2}(\Omega) ;$

$\left\langle\frac{d}{d t} \Phi_{t r}^{\prime \prime}(t), \psi\right\rangle+A\left(\Phi_{t r}^{\prime \prime}(t), \psi\right)+\int_{\Omega} I_{i o n}\left(\Phi_{t r}^{\prime \prime}(t), W^{\prime \prime}(t)\right) \psi d x=\left\langle S^{\prime \prime}(t), \psi\right\rangle \quad \forall \psi \in W^{1,2}(\Omega)$.

Then for arbitrary $\varepsilon>0$ the following estimate holds:

$\left.\left.\left|\left\langle S^{\prime}(t)-S^{\prime \prime}(t), \psi\right\rangle\right| \leqslant \frac{C}{2 \varepsilon}\left(\left\|I_{i}{ }^{\prime}(t)-I_{i}{ }^{\prime \prime}(t)\right\|_{\left(W^{1,2}(\Omega)\right.}^{2}\right)^{*}+\left\|I_{e}{ }^{\prime}(t)-I_{e}{ }^{\prime \prime}(t)\right\|_{\left(W^{1,2}(\Omega)\right.}^{2}\right)^{*}\right)+\frac{3 \varepsilon}{4}\|\psi\|_{W^{1,2}(\Omega)}^{2}$.

The constant $C>0$ does not depend on $\varepsilon, I_{i}$ and $I_{e}$ and even not on $\Phi_{0}$ and $W_{0}$.

Proof. By (2.24) and (2.25), the right-hand sides of (2.40) and (2.41) read as

$\left\langle S^{\prime}(t), \psi\right\rangle=\left\langle I_{i}^{\prime}(t), \psi\right\rangle-\int_{\Omega} \nabla \psi^{\mathrm{T}} M_{i} \nabla \bar{\psi}_{e}^{\prime} d x ; \quad\left\langle S^{\prime \prime}(t), \psi\right\rangle=\left\langle I_{i}^{\prime \prime}(t), \psi\right\rangle-\int_{\Omega} \nabla \psi^{\mathrm{T}} M_{i} \nabla \bar{\psi}_{e}^{\prime \prime} d x$

where $\bar{\psi}_{e}^{\prime}, \bar{\psi}_{e}^{\prime \prime} \in W^{1,2}(\Omega)$ are the uniquely determined solutions of the variational equations

$$
\begin{array}{ll}
\int_{\Omega} \nabla \psi^{\mathrm{T}}\left(M_{i}+M_{e}\right) \nabla \bar{\psi}_{e}^{\prime} d x=\left\langle I_{i}{ }^{\prime}+I_{e}{ }^{\prime}, \psi\right\rangle & \forall \psi \in W^{1,2}(\Omega) \text { and } \\
\int_{\Omega} \nabla \psi^{\mathrm{T}}\left(M_{i}+M_{e}\right) \nabla \bar{\psi}_{e}^{\prime \prime} d x=\left\langle I_{i}{ }^{\prime \prime}+I_{e}{ }^{\prime \prime}, \psi\right\rangle & \forall \psi \in W^{1,2}(\Omega)
\end{array}
$$

with $\int_{\Omega} \bar{\psi}_{e}^{\prime}(x, t) d x=0, \int_{\Omega} \bar{\psi}_{e}^{\prime \prime}(x, t) d x=0 \quad(\forall) t \in(0, T)$. Forming the difference of (2.44) and (2.45), we get the variational equation

$$
\int_{\Omega} \nabla \psi^{\mathrm{T}}\left(M_{i}+M_{e}\right) \nabla\left(\bar{\psi}_{e}^{\prime}-\bar{\psi}_{e}^{\prime \prime}\right) d x=\left\langle\left(I_{i}{ }^{\prime}-{I_{i}}^{\prime \prime}\right)+\left(I_{e}{ }^{\prime}-I_{e}{ }^{\prime \prime}\right), \psi\right\rangle \quad \forall \psi \in W^{1,2}(\Omega),
$$

into which $\psi=\bar{\psi}_{e}^{\prime}-\bar{\psi}_{e}^{\prime \prime}$ can be inserted as a feasible test function. Then by Assumption 2.3., 2), the Poincaré inequality may be applied to the left-hand side of (2.46) since [Evans 98], p. 275, Theorem 1, holds true together with the Rellich-Kondrachov theorem even on a bounded Lipschitz domain, cf. [ ADAMs/FourNiER 07 ] , p. 168, Theorem 6.3. This results in

$$
\begin{gathered}
C\left\|\bar{\psi}_{e}^{\prime}-\bar{\psi}_{e}^{\prime \prime}\right\|_{W^{1,2}(\Omega)}^{2} \leqslant \int_{\Omega} \nabla\left(\bar{\psi}_{e}^{\prime}-\bar{\psi}_{e}^{\prime \prime}\right)^{\mathrm{T}}\left(M_{i}+M_{e}\right) \nabla\left(\bar{\psi}_{e}^{\prime}-\bar{\psi}_{e}^{\prime \prime}\right) d x \\
\leqslant\left|\left\langle I_{i}{ }^{\prime}-I_{i}^{\prime \prime}, \bar{\psi}_{e}^{\prime}-\bar{\psi}_{e}^{\prime \prime}\right\rangle\right|+\left|\left\langle I_{e}{ }^{\prime}-I_{e}{ }^{\prime \prime}, \bar{\psi}_{e}^{\prime}-\bar{\psi}_{e}^{\prime \prime}\right\rangle\right| .
\end{gathered}
$$


For arbitrary $\varepsilon>0$, the generalized Cauchy inequality ${ }^{23)}$ implies

$$
\begin{aligned}
C\left\|\bar{\psi}_{e}^{\prime}-\bar{\psi}_{e}^{\prime \prime}\right\|_{W^{1,2}(\Omega)}^{2} \leqslant \frac{1}{2 \varepsilon}\left\|I_{i}^{\prime}(t)-I_{i}^{\prime \prime}(t)\right\|_{\left(W^{1,2}(\Omega)\right)^{*}}^{2}+\frac{\varepsilon}{2}\left\|\bar{\psi}_{e}^{\prime}-\bar{\psi}_{e}^{\prime \prime}\right\|_{W^{1,2}(\Omega)}^{2} & (2.48) \\
& +\frac{1}{2 \varepsilon}\left\|I_{e}{ }^{\prime}(t)-I_{e}^{\prime \prime}(t)\right\|_{\left(W^{1,2}(\Omega)\right)^{*}}^{2}+\frac{\varepsilon}{2}\left\|\bar{\psi}_{e}^{\prime}-\bar{\psi}_{e}^{\prime \prime}\right\|_{W^{1,2}(\Omega)}^{2},
\end{aligned}
$$

and with $\varepsilon=C / 2$, we obtain

$$
\left.\left.\frac{C}{2}\left\|\bar{\psi}_{e}^{\prime}-\bar{\psi}_{e}^{\prime \prime}\right\|_{W^{1,2}(\Omega)}^{2} \leqslant \frac{1}{C}\left(\left\|I_{i}^{\prime}(t)-I_{i}^{\prime \prime}(t)\right\|_{\left(W^{1,2}(\Omega)\right.}^{2}\right)^{*}+\left\|I_{e}{ }^{\prime}(t)-I_{e}^{\prime \prime}(t)\right\|_{\left(W^{1,2}(\Omega)\right.}^{2}\right)^{*}\right) .
$$

Further, it holds that

$$
\begin{aligned}
& \left|\left\langle S^{\prime}(t)-S^{\prime \prime}(t), \psi\right\rangle\right|=\left|\left\langle I_{i}{ }^{\prime}(t)-I_{i}^{\prime \prime}(t), \psi\right\rangle-\int_{\Omega} \nabla \psi^{\mathrm{T}} M_{i} \nabla\left(\bar{\psi}_{e}^{\prime}-\bar{\psi}_{e}^{\prime \prime}\right) d x\right| \\
\leqslant & \left|\left\langle I_{i}{ }^{\prime}(t)-I_{i}{ }^{\prime \prime}(t), \psi\right\rangle\right|+\left|\left\langle\nabla\left(\bar{\psi}_{e}^{\prime}-\bar{\psi}_{e}^{\prime \prime}\right), M_{i} \nabla \psi\right\rangle\right| \\
\leqslant & \frac{1}{2 \varepsilon}\left\|I_{i}{ }^{\prime}(t)-I_{i}^{\prime \prime}(t)\right\|_{\left(W^{1,2}(\Omega)\right)^{*}}^{2}+\frac{\varepsilon}{2}\|\psi\|_{W^{1,2}(\Omega)}^{2}+\frac{1}{2 \varepsilon^{\prime}}\left\|\bar{\psi}_{e}^{\prime}-\bar{\psi}_{e}^{\prime \prime}\right\|_{W^{1,2}(\Omega)}^{2}+\frac{\varepsilon^{\prime}}{2}\left\|M_{i}\right\|^{2} \cdot\|\psi\|_{W^{1,2}(\Omega)}^{2} \\
\leqslant & \frac{1}{2 \varepsilon}\left\|I_{i}{ }^{\prime}(t)-I_{i}^{\prime \prime}(t)\right\|_{\left(W^{1,2}(\Omega)\right)^{*}}^{2}+\frac{\varepsilon}{2}\|\psi\|_{W^{1,2}(\Omega)}^{2} \\
& \left.\quad+\frac{C}{2 \varepsilon^{\prime}}\left(\left\|I_{i}{ }^{\prime}(t)-I_{i}{ }^{\prime \prime}(t)\right\|_{\left(W^{1,2}(\Omega)\right.}^{2}\right)^{*}+\left\|I_{e}{ }^{\prime}(t)-I_{e}^{\prime \prime}(t)\right\|_{\left(W^{1,2}(\Omega)\right)^{*}}^{2}\right)+\frac{C \varepsilon^{\prime}}{2}\|\psi\|_{W^{1,2}(\Omega)}^{2}
\end{aligned}
$$

where Assumption 2.3., 2) and (2.49) have been used. Taking $\varepsilon^{\prime}=\varepsilon /(2 C)$ and equalizing the constants in the first and third term in an appropriate way, we arrive at the claimed estimate (2.42).

Lemma 2.10. For all $\varphi_{1}, \varphi_{2} \in \mathbb{R}$, the following identity holds:

$$
\begin{aligned}
\left(\varphi_{1}^{3}-(a+1) \varphi_{1}^{2}+a \varphi_{1}\right)-\left(\varphi_{2}^{3}-\right. & \left.(a+1) \varphi_{2}^{2}+a \varphi_{2}\right) \\
& =\left(\varphi_{1}-\varphi_{2}\right) \cdot\left(\varphi_{1}^{2}+\varphi_{1} \varphi_{2}+\varphi_{2}^{2}-(a+1)\left(\varphi_{1}+\varphi_{2}\right)+a\right) .
\end{aligned}
$$

Proof of Theorem 2.7. Within $(\mathrm{B})_{2}$, the Rogers-McCulloch model is specified. Assume that $\left(\Phi_{t r}{ }^{\prime}, \Phi_{e}{ }^{\prime}, W^{\prime}\right)$ and $\left(\Phi_{t r}{ }^{\prime \prime}, \Phi_{e}{ }^{\prime \prime}, W^{\prime \prime}\right)$ are weak solutions of $(\mathrm{B})_{2}$, which correspond to excitations $I_{i}{ }^{\prime}, I_{e}{ }^{\prime}, I_{i}{ }^{\prime \prime}, I_{e}{ }^{\prime \prime} \in L^{\infty}[(0, T)$, $\left.\left(W^{1,2}(\Omega)\right)\right]$ with norms bounded by $R>0$ and identical initial values $\Phi_{0}$ and $W_{0}$.

- Step 1. The difference of the reduced parabolic equations. By Theorem 2.4., 1), the pairs $\left(\Phi_{t r}{ }^{\prime}, W^{\prime}\right)$ and $\left(\Phi_{t r}{ }^{\prime \prime}, W^{\prime \prime}\right)$ satisfy for almost all $t \in[0, T]$ the equations (2.40) and (2.41) above from the reduced bidomain system. Consequently, we obtain the difference

$$
\begin{aligned}
& \left\langle\frac{d}{d t}\left(\Phi_{t r}{ }^{\prime}(t)-\Phi_{t r}{ }^{\prime \prime}(t)\right), \psi\right\rangle+A\left(\Phi_{t r}{ }^{\prime}(t)-\Phi_{t r}{ }^{\prime \prime}(t), \psi\right) \\
& \quad+\int_{\Omega}\left(I_{i o n}\left(\Phi_{t r}{ }^{\prime}(t), W^{\prime}(t)\right)-I_{i o n}\left(\Phi_{t r}{ }^{\prime \prime}(t), W^{\prime \prime}(t)\right)\right) \psi d x=\left\langle S^{\prime}(t)-S^{\prime \prime}(t), \psi\right\rangle \quad \forall \psi \in W^{1,2}(\Omega)
\end{aligned}
$$

where we may insert $\psi=\Phi_{t r}{ }^{\prime}(t)-\Phi_{t r}{ }^{\prime \prime}(t)$ as a feasible test function since $\Phi_{t r}{ }^{\prime}(t), \Phi_{t r}{ }^{\prime \prime}(t) \in W^{1,2}(\Omega)$. Using the constant $\beta>0$ from Theorem 2.4., 2), we obtain

$$
\begin{aligned}
& \frac{1}{2} \frac{d}{d t}\left\|\Phi_{t r}{ }^{\prime}(t)-\Phi_{t r}{ }^{\prime \prime}(t)\right\|_{L^{2}(\Omega)}^{2}+A\left(\Phi_{t r}{ }^{\prime}-\Phi_{t r}{ }^{\prime \prime}, \Phi_{t r}{ }^{\prime}-\Phi_{t r}{ }^{\prime \prime}\right)+\beta\left\|\Phi_{t r}{ }^{\prime}-\Phi_{t r}{ }^{\prime \prime}\right\|_{L^{2}(\Omega)}^{2} \\
& +\int_{\Omega}\left(I_{i o n}\left(\Phi_{t r}{ }^{\prime}, W^{\prime}\right)-I_{i o n}\left(\Phi_{t r}{ }^{\prime \prime}, W^{\prime \prime}\right)\right)\left(\Phi_{t r}{ }^{\prime}-\Phi_{t r}{ }^{\prime \prime}\right) d x \\
& \quad=\left\langle S^{\prime}(t)-S^{\prime \prime}(t), \Phi_{t r}{ }^{\prime}-\Phi_{t r}{ }^{\prime \prime}\right\rangle+\beta\left\|\Phi_{t r}{ }^{\prime}-\Phi_{t r}{ }^{\prime \prime}\right\|_{L^{2}(\Omega)}^{2} \Longrightarrow \quad(2.56) \\
& \frac{1}{2} \frac{d}{d t}\left\|\Phi_{t r}{ }^{\prime}-\Phi_{t r}{ }^{\prime \prime}\right\|_{L^{2}(\Omega)}^{2}+\beta\left\|\Phi_{t r}{ }^{\prime}-\Phi_{t r}{ }^{\prime \prime}\right\|_{W^{1,2}(\Omega)}^{2}+\int_{\Omega}\left(I_{i o n}\left(\Phi_{t r}{ }^{\prime}, W^{\prime}\right)-I_{i o n}\left(\Phi_{t r}{ }^{\prime \prime}, W^{\prime \prime}\right)\right)\left(\Phi_{t r}{ }^{\prime}-\Phi_{t r}{ }^{\prime \prime}\right) d x \\
& \quad \leqslant\left|\left\langle S^{\prime}(t)-S^{\prime \prime}(t), \Phi_{t r}{ }^{\prime}-\Phi_{t r}{ }^{\prime \prime}\right\rangle\right|+\beta\left\|\Phi_{t r}{ }^{\prime}-\Phi_{t r}{ }^{\prime \prime}\right\|_{L^{2}(\Omega)}^{2} . \quad(2.57)
\end{aligned}
$$

23) Cf. [Evans 98], p. 622, b. 
By Lemma 2.9., the first term on the right-hand side of (2.57) can be estimated through

$$
\begin{aligned}
& \left.\left.\left|\left\langle S^{\prime}(t)-S^{\prime \prime}(t), \Phi_{t r}{ }^{\prime}-\Phi_{t r}{ }^{\prime \prime}\right\rangle\right| \leqslant \frac{C}{2 \varepsilon_{1}}\left(\left\|I_{i}{ }^{\prime}(t)-I_{i}{ }^{\prime \prime}(t)\right\|_{\left(W^{1,2}(\Omega)\right.}^{2}\right)^{*}+\left\|I_{e}{ }^{\prime}(t)-I_{e}{ }^{\prime \prime}(t)\right\|_{\left(W^{1,2}(\Omega)\right.}^{2}\right)^{*}\right) \\
& +\frac{3 \varepsilon_{1}}{4}\left\|\Phi_{t r}^{\prime}(t)-\Phi_{t r}^{\prime \prime}(t)\right\|_{W^{1,2}(\Omega)}^{2}
\end{aligned}
$$

with a number $\varepsilon_{1}>0$ to be fixed later. Observe now that, by Lemma 2.10., the following identity holds:

$$
\begin{aligned}
\int_{\Omega}( & \left.I_{i o n}\left(\Phi_{t r}{ }^{\prime}, W^{\prime}\right)-I_{i o n}\left(\Phi_{t r}^{\prime \prime}, W^{\prime \prime}\right)\right)\left(\Phi_{t r}{ }^{\prime}-\Phi_{t r}{ }^{\prime \prime}\right) d x \\
& =\int_{\Omega}\left(\Phi_{t r}{ }^{\prime}-\Phi_{t r}{ }^{\prime \prime}\right) b\left(\left(\Phi_{t r}{ }^{\prime}\right)^{2}+\Phi_{t r}{ }^{\prime} \Phi_{t r}^{\prime \prime}+\left(\Phi_{t r}^{\prime \prime}\right)^{2}+a\right)\left(\Phi_{t r}{ }^{\prime}-\Phi_{t r}{ }^{\prime \prime}\right) d x \\
& -(a+1) b \int_{\Omega}\left(\Phi_{t r}{ }^{\prime}-\Phi_{t r}^{\prime \prime}\right)\left(\Phi_{t r}{ }^{\prime}+\Phi_{t r}^{\prime \prime}\right)\left(\Phi_{t r}{ }^{\prime}-\Phi_{t r}^{\prime \prime}\right) d x+\int_{\Omega}\left(\Phi_{t r}{ }^{\prime} W^{\prime}-\Phi_{t r}^{\prime \prime} W^{\prime \prime}\right)\left(\Phi_{t r}{ }^{\prime}-\Phi_{t r}^{\prime \prime}\right) d x .
\end{aligned}
$$

Considering further that $a, b>0$ and $\Phi_{t r}{ }^{\prime}(x, t)^{2}+\Phi_{t r}^{\prime}(x, t) \Phi_{t r}^{\prime \prime}(x, t)+\Phi_{t r}^{\prime \prime}(x, t)^{2} \geqslant 0$ for almost all $(x, t) \in \Omega_{T}$, the inequalities (2.57), (2.58) and (2.59) imply

$$
\begin{aligned}
& \frac{d}{d t}\left\|\Phi_{t r}{ }^{\prime}-\Phi_{t r}{ }^{\prime \prime}\right\|_{L^{2}(\Omega)}^{2}+2 \beta\left\|\Phi_{t r}{ }^{\prime}-\Phi_{t r}{ }^{\prime \prime}\right\|_{W^{1,2}(\Omega)}^{2} \leqslant 2 C \int_{\Omega}\left|\Phi_{t r}{ }^{\prime}-\Phi_{t r}{ }^{\prime \prime}\right| \cdot\left|\Phi_{t r}{ }^{\prime}+\Phi_{t r}{ }^{\prime \prime}\right| \cdot\left|\Phi_{t r}{ }^{\prime}-\Phi_{t r}{ }^{\prime \prime}\right| d x \\
& \left.+2 \int_{\Omega}\left(\Phi_{t r}^{\prime} W^{\prime}-\Phi_{t r}{ }^{\prime \prime} W^{\prime \prime}\right)\left(\Phi_{t r}{ }^{\prime \prime}-\Phi_{t r}{ }^{\prime}\right) d x+\frac{C}{\varepsilon_{1}}\left(\left\|I_{i}{ }^{\prime}-I_{i}{ }^{\prime \prime}\right\|_{\left(W^{1,2}(\Omega)\right.}^{2}\right)^{*}+\left\|I_{e}{ }^{\prime}-I_{e}{ }^{\prime \prime}\right\|^{2}\left(W^{1,2}(\Omega)\right)^{*}\right) \\
& +\frac{3 \varepsilon_{1}}{2}\left\|\Phi_{t r}{ }^{\prime}-\Phi_{t r}{ }^{\prime \prime}\right\|_{W^{1,2}(\Omega)}^{2}+2 \beta\left\|\Phi_{t r}{ }^{\prime}-\Phi_{t r}{ }^{\prime \prime}\right\|_{L^{2}(\Omega)}^{2} .
\end{aligned}
$$

By application of the generalized Cauchy's inequality with $\varepsilon_{2}>0$ to the first term on the right-hand side of (2.60), we get

$$
\begin{aligned}
& 2 C \int_{\Omega}\left|\Phi_{t r}^{\prime}-\Phi_{t r}^{\prime \prime}\right| \cdot\left|\Phi_{t r}^{\prime}+\Phi_{t r}^{\prime \prime}\right| \cdot\left|\Phi_{t r}^{\prime}-\Phi_{t r}^{\prime \prime}\right| d x \\
& \leqslant C \frac{\varepsilon_{2}}{2} \int_{\Omega}\left|\Phi_{t r}{ }^{\prime}+\Phi_{t r}{ }^{\prime \prime}\right|^{2} \cdot\left|\Phi_{t r}{ }^{\prime}-\Phi_{t r}{ }^{\prime \prime}\right|^{2} d x+\frac{C}{2 \varepsilon_{2}}\left\|\Phi_{t r}{ }^{\prime}-\Phi_{t r}{ }^{\prime \prime}\right\|_{L^{2}(\Omega)}^{2} \\
& \leqslant C \frac{\varepsilon_{2}}{2}\left(\int_{\Omega}\left|\Phi_{t r}{ }^{\prime}+\Phi_{t r}{ }^{\prime \prime}\right|^{4} d x\right)^{1 / 2}\left(\left\|\Phi_{t r}{ }^{\prime}-\Phi_{t r}{ }^{\prime \prime}\right\|_{L^{4}(\Omega)}^{4}\right)^{1 / 2}+\frac{C}{2 \varepsilon_{2}}\left\|\Phi_{t r}{ }^{\prime}-\Phi_{t r}{ }^{\prime \prime}\right\|_{L^{2}(\Omega)}^{2} \\
& \leqslant C \frac{\varepsilon_{2}}{2}\left(\int_{\Omega}\left|\Phi_{t r}{ }^{\prime}+\Phi_{t r}{ }^{\prime \prime}\right|^{4} d x\right)^{1 / 2}\left\|\Phi_{t r}{ }^{\prime}-\Phi_{t r}{ }^{\prime \prime}\right\|_{W^{1,2}(\Omega)}^{2}+\frac{C}{2 \varepsilon_{2}}\left\|\Phi_{t r}{ }^{\prime}-\Phi_{t r}{ }^{\prime \prime}\right\|_{L^{2}(\Omega)}^{2} .
\end{aligned}
$$

The second term on the right-hand side of (2.60) can be estimated through

$$
\begin{gathered}
2 \int_{\Omega}\left(\Phi_{t r}{ }^{\prime} W^{\prime}-\Phi_{t r}{ }^{\prime \prime} W^{\prime \prime}\right)\left(\Phi_{t r}{ }^{\prime \prime}-\Phi_{t r}{ }^{\prime}\right) d x=2 \int_{\Omega}\left(\Phi_{t r}{ }^{\prime}-\Phi_{t r}{ }^{\prime \prime}\right) W^{\prime}\left(\Phi_{t r}{ }^{\prime}-\Phi_{t r}{ }^{\prime \prime}\right) d x \\
+2 \int_{\Omega}\left(W^{\prime}-W^{\prime \prime}\right) \Phi_{t r}{ }^{\prime \prime}\left(\Phi_{t r}{ }^{\prime}-\Phi_{t r}{ }^{\prime \prime}\right) d x
\end{gathered}
$$


applying the generalized Cauchy's inequality again with $\varepsilon_{3}, \varepsilon_{4}>0$. Combining now (2.60), (2.63) and (2.67), we end up with the inequality

$$
\begin{aligned}
& \frac{d}{d t}\left\|\Phi_{t r}{ }^{\prime}-\Phi_{t r}{ }^{\prime \prime}\right\|_{L^{2}(\Omega)}^{2}+2 \beta\left\|\Phi_{t r}{ }^{\prime}-\Phi_{t r}{ }^{\prime \prime}\right\|_{W^{1,2}(\Omega)}^{2} \\
& \leqslant C \frac{\varepsilon_{2}}{2}\left(\left\|\Phi_{t r}{ }^{\prime}\right\|_{L^{4}(\Omega)}^{4}+\left\|\Phi_{t r}{ }^{\prime \prime}\right\|_{L^{4}(\Omega)}^{4}\right)^{1 / 2}\left\|\Phi_{t r}{ }^{\prime}-\Phi_{t r}{ }^{\prime \prime}\right\|_{W^{1,2}(\Omega)}^{2}+\frac{C}{2 \varepsilon_{2}}\left\|\Phi_{t r}{ }^{\prime}-\Phi_{t r}{ }^{\prime \prime}\right\|_{L^{2}(\Omega)}^{2} \\
& +C \varepsilon_{3}\left\|W^{\prime}\right\|_{L^{4}(\Omega)}^{2}\left\|\Phi_{t r}{ }^{\prime}-\Phi_{t r}{ }^{\prime \prime}\right\|_{W^{1,2}(\Omega)}^{2}+\frac{1}{\varepsilon_{3}}\left\|\Phi_{t r}{ }^{\prime}-\Phi_{t r}{ }^{\prime \prime}\right\|_{L^{2}(\Omega)}^{2} \\
& +C \varepsilon_{4}\left\|\Phi_{t r}{ }^{\prime \prime}\right\|_{L^{4}(\Omega)}^{2}\left\|\Phi_{t r}{ }^{\prime}-\Phi_{t r}{ }^{\prime \prime}\right\|_{W^{1,2}(\Omega)}^{2}+\frac{1}{\varepsilon_{4}}\left\|W^{\prime}-W^{\prime \prime}\right\|_{L^{2}(\Omega)}^{2} \\
& +\frac{C}{\varepsilon_{1}}\left(\left\|I_{i}{ }^{\prime}(t)-I_{i}{ }^{\prime \prime}(t)\right\|_{\left(W^{1,2}(\Omega)\right)^{*}}^{2}+\left\|I_{e}{ }^{\prime}(t)-I_{e}{ }^{\prime \prime}(t)\right\|_{\left(W^{1,2}(\Omega)\right)^{*}}^{2}\right) \\
& +\frac{3 \varepsilon_{1}}{2}\left\|\Phi_{t r}{ }^{\prime}-\Phi_{t r}{ }^{\prime \prime}\right\|_{W^{1,2}(\Omega)}^{2}+2 \beta\left\|\Phi_{t r}{ }^{\prime}-\Phi_{t r}{ }^{\prime \prime}\right\|_{L^{2}(\Omega)}^{2} .
\end{aligned}
$$

As a consequence of Theorem 2.6. and the assumed $L^{\infty}$-regularity of the excitations $I_{i}{ }^{\prime}, I_{e}{ }^{\prime}, I_{i}{ }^{\prime \prime}$ and $I_{e}{ }^{\prime \prime}$, the inequalities

$$
\begin{aligned}
\int_{\Omega} \Phi_{t r}{ }^{\prime}(t)^{4} d x \leqslant C\left(1+\left\|\Phi_{0}\right\|_{L^{2}(\Omega)}^{2}+\|\right. & W_{0} \|_{L^{2}(\Omega)}^{2} \\
& \left.+\left\|I^{\prime}{ }^{\prime}\right\|_{L^{\infty}\left[(0, T),\left(W^{1,2}(\Omega)\right)^{*}\right]}^{2}+\left\|I_{e}{ }^{\prime}\right\|_{L^{\infty}\left[(0, T),\left(W^{1,2}(\Omega)\right)^{*}\right]}^{2}\right) ; \\
\int_{\Omega} \Phi_{t r}{ }^{\prime \prime}(t)^{4} d x \leqslant C\left(1+\left\|\Phi_{0}\right\|_{L^{2}(\Omega)}^{2}+\|\right. & W_{0} \|_{L^{2}(\Omega)}^{2} \\
& \left.+\left\|I_{i}{ }^{\prime \prime}\right\|_{L^{\infty}\left[(0, T),\left(W^{1,2}(\Omega)\right)^{*}\right]}^{2}+\left\|I_{e}{ }^{\prime \prime}\right\|_{L^{\infty}\left[(0, T),\left(W^{1,2}(\Omega)\right)^{*}\right]}^{2}\right)
\end{aligned}
$$

hold. ${ }^{24)}$ Further, since $W^{\prime}$ is the unique (strong or weak) solution of the initial value problem (2.3), (2.6), it belongs to the space $C^{1}\left[(0, T), L^{2}(\Omega)\right] \cap C^{0}\left[[0, T], L^{2}(\Omega)\right]$ and admits the representation ${ }^{25)}$

$$
W^{\prime}(x, t)=W_{0}(x) e^{-\varepsilon t}+\varepsilon \kappa e^{-\varepsilon t} \int_{0}^{t} \Phi_{t r}^{\prime}(x, \tau) e^{\varepsilon \tau} d \tau,
$$

which implies the estimate

$$
\begin{aligned}
\int_{\Omega} W^{\prime}(t)^{4} d x \leqslant C\left\|W_{0}\right\|_{L^{4}(\Omega)}^{4}+C( & +\left\|\Phi_{0}\right\|_{L^{2}(\Omega)}^{2}+\left\|W_{0}\right\|_{L^{2}(\Omega)}^{2} \\
& \left.+\left\|I_{i}{ }^{\prime}\right\|_{L^{\infty}\left[(0, T),\left(W^{1,2}(\Omega)\right)^{*}\right]}^{2}+\left\|I_{e}{ }^{\prime}\right\|_{L^{\infty}\left[(0, T),\left(W^{1,2}(\Omega)\right)^{*}\right]}^{2}\right) .
\end{aligned}
$$

Combining finally (2.68) with (2.69), (2.70) and (2.72), we may fix the numbers $\varepsilon_{1}, \varepsilon_{2}, \varepsilon_{3}, \varepsilon_{4}>0$ in such a way that the terms with $\left\|\Phi_{t r}{ }^{\prime}-\Phi_{t r}{ }^{\prime \prime}\right\|_{W^{1,2}(\Omega)}^{2}$ will be annihilated. We arrive at

$$
\begin{aligned}
& \frac{d}{d t}\left\|\Phi_{t r}{ }^{\prime}-\Phi_{t r}{ }^{\prime \prime}\right\|_{L^{2}(\Omega)}^{2} \leqslant\left(\frac{C}{2 \varepsilon_{2}}+\frac{1}{\varepsilon_{3}}+2 \beta\right)\left\|\Phi_{t r}{ }^{\prime}-\Phi_{t r}{ }^{\prime \prime}\right\|_{L^{2}(\Omega)}^{2} \\
& +\frac{1}{\varepsilon_{4}}\left\|W^{\prime}-W^{\prime \prime}\right\|_{L^{2}(\Omega)}^{2}+\frac{C}{\varepsilon_{1}}\left(\left\|I_{i}{ }^{\prime}(t)-I_{i}^{\prime \prime}(t)\right\|_{\left(W^{1,2}(\Omega)\right)^{*}}^{2}+\left\|I_{e}{ }^{\prime}(t)-I_{e}{ }^{\prime \prime}(t)\right\|_{\left(W^{1,2}(\Omega)\right)^{*}}^{2}\right) .
\end{aligned}
$$

24) Cf. [Kunisch/Wagner 11], p. 23.

25) [WARGA 72] , p. 192, Theorem II.4.6. 
- Step 2. The difference of the gating equations. The pairs $\left(\Phi_{t r}{ }^{\prime}, W^{\prime}\right)$ and $\left(\Phi_{t r}{ }^{\prime \prime}, W^{\prime \prime}\right)$ satisfy for almost all $t \in[0, T]$ the equations

$$
\begin{array}{ll}
\left\langle\frac{d}{d t} W^{\prime}(t), \psi\right\rangle=-\int_{\Omega}\left(\varepsilon W^{\prime}(t)-\varepsilon \kappa \Phi_{t r}{ }^{\prime}(t)\right) \psi d x & \forall \psi \in L^{2}(\Omega) \text { and } \\
\left\langle\frac{d}{d t} W^{\prime \prime}(t), \psi\right\rangle=-\int_{\Omega}\left(\varepsilon W^{\prime \prime}(t)-\varepsilon \kappa \Phi_{t r}^{\prime \prime}(t)\right) \psi d x & \forall \psi \in L^{2}(\Omega) .
\end{array}
$$

Inserting the feasible test function $\psi=W^{\prime}(t)-W^{\prime \prime}(t)$ into the difference of (2.74) and (2.75), we get

$$
\begin{aligned}
& \left\langle\frac{d}{d t}\left(W^{\prime}(t)-W^{\prime \prime}(t)\right), W^{\prime}(t)-W^{\prime \prime}(t)\right\rangle \\
& =-\varepsilon \int_{\Omega}\left(W^{\prime}-W^{\prime \prime}\right)^{2} d x+\varepsilon \kappa \int_{\Omega}\left(\Phi_{t r}^{\prime}-\Phi_{t r}{ }^{\prime \prime}\right)\left(W^{\prime}-W^{\prime \prime}\right) d x \Longrightarrow \\
& \frac{d}{d t}\left(\left\|W^{\prime}-W^{\prime \prime}\right\|_{L^{2}(\Omega)}^{2}\right) \leqslant(2 \varepsilon+\varepsilon \kappa)\left\|W^{\prime}-W^{\prime \prime}\right\|_{L^{2}(\Omega)}^{2}+\varepsilon \kappa\left\|\Phi_{t r}{ }^{\prime}-\Phi_{t r}{ }^{\prime \prime}\right\|_{L^{2}(\Omega)}^{2} .
\end{aligned}
$$

- Step 3. The estimates for the differences $\left\|\Phi_{t r}{ }^{\prime}-\Phi_{t r}{ }^{\prime \prime}\right\|_{L^{\infty}\left[(0, T), L^{2}(\Omega)\right.}^{2},\left\|W^{\prime}-W^{\prime \prime}\right\|_{L^{2}\left(\Omega_{T}\right)}^{2}$ and $\left\|W^{\prime}-W^{\prime \prime}\right\|_{L^{\infty}}^{2}\left[(0, T), L^{2}(\Omega)\right]$. After equalizing of the constants on the right-hand sides, the inequalities (2.73) and (2.77) give together

$$
\begin{array}{r}
\frac{d}{d t}\left(\left\|\Phi_{t r}{ }^{\prime}-\Phi_{t r}{ }^{\prime \prime}\right\|_{L^{2}(\Omega)}^{2}+\left\|W^{\prime}-W^{\prime \prime}\right\|_{L^{2}(\Omega)}^{2}\right) \\
+\frac{C}{\varepsilon_{1}}\left(\left\|I_{i}{ }^{\prime}(t)-I_{i}{ }^{\prime \prime}(t)\right\|_{\left(W^{1,2}(\Omega)\right.}{ }^{2}{ }^{*}+\left\|I_{e}{ }^{\prime}(t)-I_{e}{ }^{\prime \prime}(t)\right\|_{\left(W^{1,2}(\Omega)\right)^{*}}{ }^{*}\right),
\end{array}
$$

By application of Gronwall's inequality, ${ }^{26)}(2.78)$ implies

$$
\begin{aligned}
& \left\|\Phi_{t r}{ }^{\prime}(t)-\Phi_{t r}{ }^{\prime \prime}(t)\right\|_{L^{2}(\Omega)}^{2}+\left\|W^{\prime}(t)-W^{\prime \prime}(t)\right\|_{L^{2}(\Omega)}^{2} \leqslant e^{C t}\left(\left\|\Phi_{t r}{ }^{\prime}(0)-\Phi_{t r}{ }^{\prime \prime}(0)\right\|_{L^{2}(\Omega)}^{2}\right. \\
& \left.\quad+\left\|W^{\prime}(0)-W^{\prime \prime}(0)\right\|_{L^{2}(\Omega)}^{2}+\frac{C}{\varepsilon_{1}} \int_{0}^{t}\left(\left\|I_{i}{ }^{\prime}(\tau)-I_{i}^{\prime \prime}(\tau)\right\|_{\left(W^{1,2}(\Omega)\right)^{*}}^{2}+\left\|I_{e}{ }^{\prime}(\tau)-I_{e}{ }^{\prime \prime}(\tau)\right\|_{\left(W^{1,2}(\Omega)\right)^{*}}^{2}\right) d \tau\right) \\
& \quad \leqslant e^{C T} \frac{C}{\varepsilon_{1}}\left(\left\|I_{i}{ }^{\prime}-I_{i}{ }^{\prime \prime}\right\|_{L^{2}\left[(0, T),\left(W^{1,2}(\Omega)\right)^{*}\right]}^{2}+\left\|I_{e}{ }^{\prime}-I_{e}{ }^{\prime \prime}\right\|_{L^{2}\left[(0, T),\left(W^{1,2}(\Omega)\right)^{*}\right]}^{2}\right) .
\end{aligned}
$$

From the last inequality, we get the desired estimates:

$$
\begin{gathered}
\left\|\Phi_{t r}{ }^{\prime}-\Phi_{t r}{ }^{\prime \prime}\right\|_{L^{\infty}\left[(0, T), L^{2}(\Omega)\right]}^{2} \leqslant e^{C T} \frac{C}{\varepsilon_{1}}\left(\left\|I_{i}{ }^{\prime}-I_{i}{ }^{\prime \prime}\right\|_{L^{2}\left[(0, T),\left(W^{1,2}(\Omega)\right)^{*}\right]}^{2}\right. \\
\left.\left.+\left\|I_{e}{ }^{\prime}-I_{e}{ }^{\prime \prime}\right\|_{L^{2}\left[(0, T),\left(W^{1,2}(\Omega)\right)^{*}\right]}^{2}\right]\right) \\
\begin{array}{c}
\left\|W^{\prime}-W^{\prime \prime}\right\|_{L^{\infty}\left[(0, T), L^{2}(\Omega)\right]}^{2} \leqslant e^{C T} \frac{C}{\varepsilon_{1}}\left(\left\|I_{i}{ }^{\prime}-I_{i}{ }^{\prime \prime}\right\|_{L^{2}\left[(0, T),\left(W^{1,2}(\Omega)\right)^{*}\right]}^{2}\right. \\
\left.+\left\|I_{e}{ }^{\prime}-I_{e}{ }^{\prime \prime}\right\|_{L^{2}\left[(0, T),\left(W^{1,2}(\Omega)\right)^{*}\right]}^{2}\right)
\end{array} \\
\begin{array}{c}
\left\|W^{\prime}-W^{\prime \prime}\right\|_{L^{2}\left(\Omega_{T}\right)}^{2} \leqslant T e^{C T} \frac{C}{\varepsilon_{1}}\left(\left\|I_{i}{ }^{\prime}-I_{i}^{\prime \prime}\right\|_{L^{2}\left[(0, T),\left(W^{1,2}(\Omega)\right)^{*}\right]}^{2}\right. \\
\left.+\left\|I_{e}{ }^{\prime}-I_{e}{ }^{\prime \prime}\right\|_{L^{2}\left[(0, T),\left(W^{1,2}(\Omega)\right)^{*}\right]}^{2}\right]
\end{array}
\end{gathered}
$$

26)

[EVANS 98], p. 624, j. 
- Step 4. The estimate for the difference $\left\|\Phi_{t r}{ }^{\prime}-\Phi_{t r}{ }^{\prime \prime}\right\|_{L^{2}\left[(0, T), W^{1,2}(\Omega)\right]}^{2}$. We go back to $(2.68)-(2.72)$ and choose the numbers $\varepsilon_{1}, \ldots, \varepsilon_{4}>0$ alternatively in such a way that

$$
\begin{aligned}
& \frac{d}{d t}\left\|\Phi_{t r}{ }^{\prime}-\Phi_{t r}{ }^{\prime \prime}\right\|_{L^{2}(\Omega)}^{2}+\beta\left\|\Phi_{t r}{ }^{\prime}-\Phi_{t r}{ }^{\prime \prime}\right\|_{W^{1,2}(\Omega)}^{2} \leqslant\left(\frac{C}{2 \varepsilon_{2}}+\frac{1}{\varepsilon_{3}}+2 \beta\right)\left\|\Phi_{t r}{ }^{\prime}-\Phi_{t r}{ }^{\prime \prime}\right\|_{L^{2}(\Omega)}^{2} \\
& \left.+\frac{1}{\varepsilon_{4}}\left\|W^{\prime}-W^{\prime \prime}\right\|_{L^{2}(\Omega)}^{2}+\frac{C}{\varepsilon_{1}}\left(\left\|I_{i}{ }^{\prime}(t)-I_{i}{ }^{\prime \prime}(t)\right\|_{\left(W^{1,2}(\Omega)\right.}^{2}\right)^{*}+\left\|I_{e}{ }^{\prime}(t)-I_{e}{ }^{\prime \prime}(t)\right\|_{\left(W^{1,2}(\Omega)\right)^{*}}^{2}\right) .
\end{aligned}
$$

This leads to the following modification of (2.78):

$$
\begin{aligned}
& \frac{d}{d t}\left(\left\|\Phi_{t r}{ }^{\prime}-\Phi_{t r}{ }^{\prime \prime}\right\|_{L^{2}(\Omega)}^{2}+\left\|W^{\prime}-W^{\prime \prime}\right\|_{L^{2}(\Omega)}^{2}\right)+\beta\left\|\Phi_{t r}{ }^{\prime}-\Phi_{t r}{ }^{\prime \prime}\right\|_{W^{1,2}(\Omega)}^{2} \leqslant C \cdot\left(\left\|\Phi_{t r}{ }^{\prime}-\Phi_{t r}{ }^{\prime \prime}\right\|_{L^{2}(\Omega)}^{2}\right. \\
& \left.\left.\left.+\left\|W^{\prime}-W^{\prime \prime}\right\|_{L^{2}(\Omega)}^{2}\right)+\frac{C}{\varepsilon_{1}}\left(\left\|I_{i}{ }^{\prime}(t)-I_{i}^{\prime \prime}(t)\right\|_{\left(W^{1,2}(\Omega)\right.}^{2}\right)^{*}+\left\|I_{e}{ }^{\prime}(t)-I_{e}{ }^{\prime \prime}(t)\right\|_{\left(W^{1,2}(\Omega)\right.}^{2}\right)^{*}\right) .
\end{aligned}
$$

Together with (2.80), we arrive at

$\left\|\Phi_{t r}{ }^{\prime}(t)-\Phi_{t r}{ }^{\prime \prime}(t)\right\|_{W^{1,2}(\Omega)}^{2} \leqslant C\left(\left\|I_{i}{ }^{\prime}-I_{i}^{\prime \prime}\right\|_{L^{2}\left[(0, T),\left(W^{1,2}(\Omega)\right)^{*}\right]}^{2}+\left\|I_{e}{ }^{\prime}-I_{e}{ }^{\prime \prime}\right\|_{L^{2}\left[(0, T),\left(W^{1,2}(\Omega)\right)^{*}\right]}^{2}\right)$,

which implies

$$
\begin{aligned}
\left\|\Phi_{t r}{ }^{\prime}-\Phi_{t r}{ }^{\prime \prime}\right\|_{L^{2}\left[(0, T), W^{1,2}(\Omega)\right]}^{2} \leqslant C\left(\left\|I_{i}{ }^{\prime}-I_{i}{ }^{\prime \prime}\right\|_{L^{2}[(0, T)}^{2},\left(W^{1,2}(\Omega)\right)^{*}\right] \\
\left.+\left\|I_{e}{ }^{\prime}-I_{e}^{\prime \prime}\right\|_{L^{2}\left[(0, T),\left(W^{1,2}(\Omega)\right)^{*}\right]}^{2}\right) .
\end{aligned}
$$

- Step 5. The estimate for the difference $\left\|W^{\prime}-W^{\prime \prime}\right\|_{W^{1,2}\left[(0, T), L^{2}(\Omega)\right.}^{2}$. Into the difference of $(2.74)$ and (2.75), we insert the test function $\psi=\left(\partial W^{\prime}(t) / \partial t\right)-\left(\partial W^{\prime \prime}(t) / \partial t\right)$, which obviously belongs to $L^{2}\left(\Omega_{T}\right)$ and is therefore admissible. Then we get with the generalized Cauchy's inequality

$$
\begin{aligned}
\left\|\frac{\partial W^{\prime}}{\partial t}-\frac{\partial W^{\prime \prime}}{\partial t}\right\|_{L^{2}(\Omega)}^{2} \leqslant \varepsilon \frac{\varepsilon_{5}}{2}\left\|\frac{\partial W^{\prime}}{\partial t}-\frac{\partial W^{\prime \prime}}{\partial t}\right\|_{L^{2}(\Omega)}^{2} & +\frac{\varepsilon}{2 \varepsilon_{5}}\left\|W^{\prime}-W^{\prime \prime}\right\|_{L^{2}(\Omega)}^{2} \\
& +\varepsilon \kappa \frac{\varepsilon_{6}}{2}\left\|\frac{\partial W^{\prime}}{\partial t}-\frac{\partial W^{\prime \prime}}{\partial t}\right\|_{L^{2}(\Omega)}^{2}+\frac{\varepsilon \kappa}{2 \varepsilon_{6}}\left\|\Phi_{t r}{ }^{\prime}-\Phi_{t r}{ }^{\prime \prime}\right\|_{L^{2}(\Omega)}^{2}
\end{aligned}
$$

for arbitrary $\varepsilon_{5}, \varepsilon_{6}>0$. Fixing the numbers $\varepsilon_{5}$ and $\varepsilon_{6}$ in such a way that $\varepsilon \varepsilon_{5}+\varepsilon \kappa \varepsilon_{6}=1$, we find together with (2.81) and (2.82):

$$
\begin{gathered}
\begin{array}{c}
\left\|\frac{\partial W^{\prime}}{\partial t}-\frac{\partial W^{\prime \prime}}{\partial t}\right\|_{L^{2}(\Omega)}^{2} \leqslant \frac{\varepsilon}{\varepsilon_{5}}\left\|W^{\prime}-W^{\prime \prime}\right\|_{L^{2}(\Omega)}^{2}+\frac{\varepsilon \kappa}{\varepsilon_{6}}\left\|\Phi_{t r}{ }^{\prime}-\Phi_{t r}{ }^{\prime \prime}\right\|_{L^{2}(\Omega)}^{2} \\
\leqslant C\left(\left\|I_{i}{ }^{\prime}-I_{i}{ }^{\prime \prime}\right\|_{L^{2}\left[(0, T),\left(W^{1,2}(\Omega)\right)^{*}\right]}^{2}+\left\|I_{e}{ }^{\prime}-I_{e}{ }^{\prime \prime}\right\|_{L^{2}\left[(0, T),\left(W^{1,2}(\Omega)\right)^{*}\right]}^{2}\right)
\end{array} \\
\begin{array}{c}
\left\|\frac{\partial W^{\prime}}{\partial t}-\frac{\partial W^{\prime \prime}}{\partial t}\right\|_{L^{2}\left[(0, T), L^{2}(\Omega)\right]}^{2} \leqslant C T\left(\left\|I_{i}{ }^{\prime}-I_{i}{ }^{\prime \prime}\right\|_{L^{2}\left[(0, T),\left(W^{1,2}(\Omega)\right)^{*}\right]}^{2}\right. \\
\left.+\left\|I_{e}{ }^{\prime}-I_{e}{ }^{\prime \prime}\right\|_{L^{2}\left[(0, T),\left(W^{1,2}(\Omega)\right)^{*}\right]}^{2}\right),
\end{array}
\end{gathered}
$$

and with (2.83), we get the estimate

$$
\begin{aligned}
\left\|W^{\prime}-W^{\prime \prime}\right\|_{W^{1,2}\left[(0, T), L^{2}(\Omega)\right]}^{2} \leqslant C\left(\left\|I_{i}{ }^{\prime}-I_{i}{ }^{\prime \prime}\right\|_{L^{2}\left[(0, T),\left(W^{1,2}(\Omega)\right)^{*}\right]}^{2}\right. & (2.91) \\
& \left.+\left\|I_{e}{ }^{\prime}-I_{e}{ }^{\prime \prime}\right\|_{L^{2}\left[(0, T),\left(W^{1,2}(\Omega)\right)^{*}\right]}^{2}\right) .
\end{aligned}
$$


- Step 6. The estimate for the difference $\left\|\Phi_{t r}{ }^{\prime}-\Phi_{t r}{ }^{\prime \prime}\right\|_{W^{1,4 / 3}}\left[(0, T),\left(W^{1,2}(\Omega)\right)^{*}\right]$. We will exploit the definition of the dual norm where $q=4 / 3$. Then (2.55) yields

$$
\begin{aligned}
& \left.\int_{0}^{T}\left\|\frac{\partial \Phi_{t r}{ }^{\prime}(t)}{\partial t}-\frac{\partial \Phi_{t r}{ }^{\prime \prime}(t)}{\partial t}\right\|_{\left(W^{1,2}(\Omega)\right.}^{q}\right)^{*} d t=\int_{0}^{T} \sup _{\|\|_{W^{1,2}(\Omega)}=1}\left|\left\langle\frac{\partial \Phi_{t r}{ }^{\prime}}{\partial t}-\frac{\partial \Phi_{t r}{ }^{\prime \prime}}{\partial t}, \psi\right\rangle\right|^{q} d t \\
& \leqslant C \cdot \int_{0}^{T}\left(\sup _{\ldots}\left|\left\langle S^{\prime}(t)-S^{\prime \prime}(t), \psi\right\rangle\right|^{q}+\sup _{\ldots}\left|A\left(\Phi_{t r}{ }^{\prime}-\Phi_{t r}{ }^{\prime \prime}, \psi\right)\right|^{q}\right. \\
& +\sup _{\ldots} \mid \int_{\Omega}\left(I_{i o n}\left(\Phi_{t r}{ }^{\prime}, W^{\prime}\right)-\left.I_{\text {ion }}\left(\Phi_{t r}{ }^{\prime \prime}, W^{\prime \prime}\right) \psi d x\right|^{q}\right) d t \quad \Longrightarrow \\
& \left(\int_{0}^{T}\left\|\frac{\partial \Phi_{t r}{ }^{\prime}}{\partial t}-\frac{\partial \Phi_{t r}^{\prime \prime}}{\partial t}\right\|_{\left(W^{1,2}(\Omega)\right)^{*}}^{q} d t\right)^{1 / q} \\
& \leqslant C \cdot\left(\int_{0}^{T} \sup _{\ldots}\left|\left\langle S^{\prime}(t)-S^{\prime \prime}(t), \psi\right\rangle\right|^{2} d t\right)^{1 / 2}+C \cdot\left(\int_{0}^{T} \sup _{\ldots}\left|A\left(\Phi_{t r}{ }^{\prime}-\Phi_{t r}{ }^{\prime \prime}, \psi\right)\right|^{2} d t\right)^{1 / 2} \\
& +C \cdot\left(\int_{0}^{T}\left(\sup _{\ldots}\left\|I_{i o n}\left(\Phi_{t r}{ }^{\prime}, W^{\prime}\right)-I_{i o n}\left(\Phi_{t r}{ }^{\prime \prime}, W^{\prime \prime}\right)\right\|_{L^{q}(\Omega)} \cdot\|\psi\|_{L^{p}(\Omega)}\right)^{q} d t\right)^{1 / q} .
\end{aligned}
$$

We estimate the three terms on the right-hand side of (2.94) separately. For the first term, we get from (2.51)

$$
\begin{aligned}
& \sup _{\|\psi\|_{W^{1,2}(\Omega)}}\left|\left\langle S^{\prime}(t)-S^{\prime \prime}(t), \psi\right\rangle\right|^{2} \leqslant C\left(\sup _{\ldots}\left|\left\langle I_{i}^{\prime}(t)-I_{i}^{\prime \prime}(t), \psi\right\rangle\right|^{2}+\underset{\ldots}{\sup }\left|\left\langle\nabla\left(\bar{\psi}_{e}^{\prime}-\bar{\psi}_{e}^{\prime \prime}\right), M_{i} \nabla \psi\right\rangle\right|^{2}\right) \\
& \left.\leqslant C\left(\left\|I_{i}^{\prime}(t)-I_{i}^{\prime \prime}(t)\right\|_{\left(W^{1,2}(\Omega)\right.}^{2}\right)^{*}+\left\|M_{i}\right\|^{2} \cdot\left\|\bar{\psi}_{e}^{\prime}-\bar{\psi}_{e}^{\prime \prime}\right\|_{W^{1,2}(\Omega)}^{2}\right)
\end{aligned}
$$

and with (2.49), we arrive at

$$
\begin{aligned}
& \sup _{\ldots}\left|\left\langle S^{\prime}(t)-S^{\prime \prime}(t), \psi\right\rangle\right|^{2} \leqslant C\left(\left\|I_{i}{ }^{\prime}(t)-I_{i}{ }^{\prime \prime}(t)\right\|_{\left(W^{1,2}(\Omega)\right)^{*}}^{2}+\left\|I_{e}{ }^{\prime}(t)-I_{e}{ }^{\prime \prime}(t)\right\|_{\left(W^{1,2}(\Omega)\right.}^{2}\right) \\
& \left(\int_{0}^{T} \sup _{\ldots}\left|\left\langle\frac{1}{1+\lambda}\left(\lambda\left(I_{i}{ }^{\prime}(t)-I_{i}{ }^{\prime \prime}(t)\right)-\left(I_{e}{ }^{\prime}(t)-I_{e}{ }^{\prime \prime}(t)\right)\right), \psi\right\rangle\right|^{2} d t\right)^{1 / 2} \\
& \quad \leqslant C\left(\left\|I_{i}{ }^{\prime}-I_{i}{ }^{\prime \prime}\right\|_{L^{2}\left[(0, T),\left(W^{1,2}(\Omega)\right)^{*}\right]}^{2}+\left\|I_{e}{ }^{\prime}-I_{e}{ }^{\prime \prime}\right\|_{L^{2}\left[(0, T),\left(W^{1,2}(\Omega)\right)^{*}\right]}^{2}\right) .
\end{aligned}
$$

For the second term, we obtain from Theorem 2.4., 2), and (2.87):

$$
\begin{aligned}
& \left|A\left(\Phi_{t r}{ }^{\prime}-\Phi_{t r}{ }^{\prime \prime}, \psi\right)\right|^{2} \leqslant \gamma^{2}\left\|\Phi_{t r}{ }^{\prime}-\Phi_{t r}{ }^{\prime \prime}\right\|_{W^{1,2}(\Omega)}^{2} \cdot\|\psi\|_{W^{1,2}(\Omega)}^{2} \Longrightarrow \\
& \left.\left(\int_{0}^{T} \sup _{\cdots}\left|M\left(\Phi_{t r}{ }^{\prime}-\Phi_{t r}{ }^{\prime \prime}, \psi\right)\right|^{2} d t\right)^{1 / 2} \leqslant C\left\|\Phi_{t r}{ }^{\prime}-\Phi_{t r}{ }^{\prime \prime}\right\|_{L^{2}\left[(0, T), W^{1,2}(\Omega)\right.}\right] \\
& \leqslant C\left(\left\|I_{i}{ }^{\prime}-I_{i}{ }^{\prime \prime}\right\|_{L^{2}\left[(0, T),\left(W^{1,2}(\Omega)\right)^{*}\right]}^{2}+\left\|I_{e}{ }^{\prime}-I_{e}{ }^{\prime \prime}\right\|_{L^{2}\left[(0, T),\left(W^{1,2}(\Omega)\right)^{*}\right]}^{2}\right) .
\end{aligned}
$$

By Lemma 2.10., the integrand within the third term may be rewritten as follows:

$$
\begin{aligned}
& \left\|I_{i o n}\left(\Phi_{t r}{ }^{\prime}, W^{\prime}\right)-I_{i o n}\left(\Phi_{t r}{ }^{\prime \prime}, W^{\prime \prime}\right)\right\|_{L^{q}(\Omega)} \\
& \leqslant\left\|b\left(\left(\Phi_{t r}{ }^{\prime}\right)^{2}+\Phi_{t r}{ }^{\prime} \Phi_{t r}{ }^{\prime \prime}+\left(\Phi_{t r}{ }^{\prime \prime}\right)^{2}-(a+1)\left(\Phi_{t r}{ }^{\prime}+\Phi_{t r}{ }^{\prime \prime}\right)+a\right)\left(\Phi_{t r}{ }^{\prime}-\Phi_{t r}{ }^{\prime \prime}\right)\right\|_{L^{q}(\Omega)} \\
& +\left\|\left(\Phi_{t r}{ }^{\prime}-\Phi_{t r}{ }^{\prime \prime}\right) W^{\prime}\right\|_{L^{q}(\Omega)}+\left\|\left(W^{\prime}-W^{\prime \prime}\right) \Phi_{t r}{ }^{\prime \prime}\right\|_{L^{q}(\Omega)}=J_{1}+J_{2}+J_{3} .
\end{aligned}
$$


For $J_{1}$, we get with $q=4 / 3$

$$
\begin{gathered}
J_{1}=\left(\int_{\Omega} b^{q}\left(\left(\Phi_{t r}{ }^{\prime}\right)^{2}+\Phi_{t r}{ }^{\prime} \Phi_{t r}{ }^{\prime \prime}+\left(\Phi_{t r}{ }^{\prime \prime}\right)^{2}-(a+1)\left(\Phi_{t r}{ }^{\prime}+\Phi_{t r}{ }^{\prime \prime}\right)+a\right)^{q}\left(\Phi_{t r}{ }^{\prime}-\Phi_{t r}{ }^{\prime \prime}\right)^{q} d x\right)^{1 / q} \\
\leqslant\left(\int _ { \Omega } b ^ { 3 q / 2 } \left(\left(\Phi_{t r}{ }^{\prime}\right)^{2}+\Phi_{t r}{ }^{\prime} \Phi_{t r}{ }^{\prime \prime}+\left(\Phi_{t r}{ }^{\prime \prime}\right)^{2}\right.\right. \\
\left.\left.\quad-(a+1)\left(\Phi_{t r}{ }^{\prime}+\Phi_{t r}{ }^{\prime \prime}\right)+a\right)^{3 q / 2} d x\right)^{2 / 3 q}\left(\int_{\Omega}\left(\Phi_{t r}{ }^{\prime}-\Phi_{t r}{ }^{\prime \prime}\right)^{3 q}\right)^{1 / 3 q} \\
\leqslant C\left(1+\left\|\Phi_{t r}{ }^{\prime}(t)\right\|_{L^{4}(\Omega)}^{4}+\left\|\Phi_{t r}{ }^{\prime \prime}(t)\right\|_{L^{4}(\Omega)}^{4}\right)^{2} \cdot\left\|\Phi_{t r}{ }^{\prime}-\Phi_{t r}{ }^{\prime \prime}\right\|_{W^{1,2}(\Omega)}
\end{gathered}
$$

due to the continuous imbedding $W^{1,2}(\Omega) \hookrightarrow L^{4}(\Omega)$. Further, using $(2.72)$ and the boundedness of the norms of $I_{i}{ }^{\prime}$ and $I_{e}{ }^{\prime}, J_{2}$ may be estimated through

$$
\begin{aligned}
& J_{2}=\left(\int_{\Omega}\left(\Phi_{t r}{ }^{\prime}-\Phi_{t r}{ }^{\prime \prime}\right)^{q}\left(W^{\prime}\right)^{q} d x\right)^{1 / q} \leqslant\left(\int_{\Omega}\left(\Phi_{t r}{ }^{\prime}-\Phi_{t r}{ }^{\prime \prime}\right)^{2} d x\right)^{2 / 3 q}\left(\int_{\Omega}\left(W^{\prime}\right)^{4} d x\right)^{1 / 3 q} \\
& =\left(\left\|\Phi_{t r}{ }^{\prime}-\Phi_{t r}{ }^{\prime \prime}\right\|_{L^{2}(\Omega)}^{4 / 3} \cdot\left\|W^{\prime}\right\|_{L^{4}(\Omega)}^{4 / 3}\right)^{3 / 4}=\left\|\Phi_{t r}{ }^{\prime}-\Phi_{t r}{ }^{\prime \prime}\right\|_{L^{2}(\Omega)} \cdot\left\|W^{\prime}\right\|_{L^{4}(\Omega)} \leqslant C\left\|\Phi_{t r}{ }^{\prime}-\Phi_{t r}{ }^{\prime \prime}\right\|_{L^{2}(\Omega)}
\end{aligned}
$$

Finally, (2.70) allows to estimate $J_{3}$ through

$$
\begin{aligned}
& J_{3}=\left(\int_{\Omega}\left(W^{\prime}-W^{\prime \prime}\right)^{q}\left(\Phi_{t r}{ }^{\prime \prime}\right)^{q} d x\right)^{1 / q} \leqslant\left(\int_{\Omega}\left(W^{\prime}-W^{\prime \prime}\right)^{2} d x\right)^{2 / 3 q}\left(\int_{\Omega}\left(\Phi_{t r}{ }^{\prime \prime}\right)^{4} d x\right)^{1 / 3 q} \\
& =\left(\left\|W^{\prime}-W^{\prime \prime}\right\|_{L^{2}(\Omega)}^{4 / 3} \cdot\left\|\Phi_{t r}{ }^{\prime \prime}\right\|_{L^{4}(\Omega)}^{4 / 3}\right)^{3 / 4}=\left\|W^{\prime}-W^{\prime \prime}\right\|_{L^{2}(\Omega)} \cdot\left\|\Phi_{t r}{ }^{\prime \prime}\right\|_{L^{4}(\Omega)} \leqslant C\left\|W^{\prime}-W^{\prime \prime}\right\|_{L^{2}(\Omega)} .
\end{aligned}
$$

The estimates (2.105), (2.107) and (2.109) together imply that

$$
\begin{gathered}
\left.\left(\int_{0}^{T} \underset{\ldots}{(} \underset{\sup }{\|} I_{\text {ion }}\left(\Phi_{t r}{ }^{\prime}, W^{\prime}\right)-I_{\text {ion }}\left(\Phi_{t r}{ }^{\prime \prime}, W^{\prime \prime}\right)\left\|_{L^{4 / 3}(\Omega)} \cdot\right\| \psi \|_{L^{4}(\Omega)}\right)^{4 / 3} d t\right)^{3 / 4} \\
\leqslant C \cdot\left(\int _ { 0 } ^ { T } \left(\left(1+\left\|\Phi_{t r}{ }^{\prime}(t)\right\|_{L^{4}(\Omega)}^{4}+\left\|\Phi_{t r}{ }^{\prime \prime}(t)\right\|_{L^{4}(\Omega)}^{4}\right)^{2} \cdot\left\|\Phi_{t r}{ }^{\prime}-\Phi_{t r}{ }^{\prime \prime}\right\|_{W^{1,2}(\Omega)}\right.\right. \\
\left.\left.\quad+\left\|\Phi_{t r}{ }^{\prime}-\Phi_{t r}{ }^{\prime \prime}\right\|_{L^{2}(\Omega)}+\left\|W^{\prime}-W^{\prime \prime}\right\|_{L^{2}(\Omega)}\right)^{4 / 3} d t\right)^{3 / 4} \\
\leqslant C \cdot\left(\int_{0}^{T}\left(1+\left\|\Phi_{t r}{ }^{\prime}(t)\right\|_{L^{4}(\Omega)}^{4}+\left\|\Phi_{t r}{ }^{\prime \prime}(t)\right\|_{L^{4}(\Omega)}^{4}\right)^{8 / 3} \cdot\left\|\Phi_{t r}{ }^{\prime}-\Phi_{t r}{ }^{\prime \prime}\right\|_{W^{1,2}(\Omega)}^{4 / 3}\right. \\
\left.\quad+\int_{0}^{T}\left\|\Phi_{t r}{ }^{\prime}-\Phi_{t r}{ }^{\prime \prime}\right\|_{W^{1,2}(\Omega)}^{4 / 3} d t+\int_{0}^{T}\left\|W^{\prime}-W^{\prime \prime}\right\|_{L^{2}(\Omega)}^{4 / 3} d t\right)^{3 / 4} .
\end{gathered}
$$

With (2.69), (2.70), (2.80) and (2.86), we obtain

$$
\begin{aligned}
& \left.\left(\int_{0}^{T} \underset{\ldots}{ } \underset{\ldots}{ } \sup \left\|I_{\text {ion }}\left(\Phi_{t r}{ }^{\prime}, W^{\prime}\right)-I_{\text {ion }}\left(\Phi_{t r}{ }^{\prime \prime}, W^{\prime \prime}\right)\right\|_{L^{4 / 3}(\Omega)} \cdot\|\psi\|_{L^{4}(\Omega)}\right)^{4 / 3} d t\right)^{3 / 4} \\
& \quad \leqslant 3 C \cdot\left(\int_{0}^{T}\left(\left\|I_{i}{ }^{\prime}-I_{i}^{\prime \prime}\right\|_{L^{2}\left[(0, T),\left(W^{1,2}(\Omega)\right)^{*}\right]}^{2}+\left\|I_{e}{ }^{\prime}-I_{e}{ }^{\prime \prime}\right\|_{L^{2}\left[(0, T),\left(W^{1,2}(\Omega)\right)^{*}\right]}^{2}\right)^{2 / 3} d t\right)^{3 / 4} \\
& \quad \leqslant C \cdot \operatorname{Max}\left(\left\|I_{i}{ }^{\prime}-I_{i}{ }^{\prime \prime}\right\|_{L^{2}\left[(0, T),\left(W^{1,2}(\Omega)\right)^{*}\right]},\left\|I_{e}{ }^{\prime}-I_{e}{ }^{\prime \prime}\right\|_{L^{2}\left[(0, T),\left(W^{1,2}(\Omega)\right)^{*}\right]}\right) .
\end{aligned}
$$

Summing up, we get from (2.97), (2.100) and (2.114)

$$
\begin{aligned}
& \left\|\frac{\partial \Phi_{t r}{ }^{\prime}}{\partial t}-\frac{\partial \Phi_{t r}{ }^{\prime \prime}}{\partial t}\right\|_{L^{4 / 3}\left[(0, T),\left(W^{1,2}(\Omega)\right)^{*}\right]} \leqslant C \cdot \operatorname{Max}\left(\left\|I_{i}{ }^{\prime}-I_{i}{ }^{\prime \prime}\right\|_{L^{2}\left[(0, T),\left(W^{1,2}(\Omega)\right)^{*}\right]},\right. \\
& \left.\left\|I_{e}{ }^{\prime}-I_{e}{ }^{\prime \prime}\right\|_{L^{2}\left[(0, T),\left(W^{1,2}(\Omega)\right)^{*}\right]},\left\|I_{i}{ }^{\prime}-I_{i}{ }^{\prime \prime}\right\|_{L^{2}\left[(0, T),\left(W^{1,2}(\Omega)\right)^{*}\right]}^{2},\left\|I_{e}{ }^{\prime}-I_{e}{ }^{\prime \prime}\right\|_{L^{2}\left[(0, T),\left(W^{1,2}(\Omega)\right)^{*}\right]}^{2}\right)
\end{aligned}
$$


and, considering (2.81), we arrive finally at

$$
\begin{aligned}
& \left\|\Phi_{t r}{ }^{\prime}-\Phi_{t r}{ }^{\prime \prime}\right\|_{W^{1,4 / 3}\left[(0, T),\left(W^{1,2}(\Omega)\right)^{*}\right]} \leqslant C \cdot \operatorname{Max}\left(\left\|I_{i}{ }^{\prime}-I_{i}{ }^{\prime \prime}\right\|_{L^{2}\left[(0, T),\left(W^{1,2}(\Omega)\right)^{*}\right]},\right. \\
& \left.\left\|I_{e}{ }^{\prime}-I_{e}{ }^{\prime \prime}\right\|_{L^{2}\left[(0, T),\left(W^{1,2}(\Omega)\right)^{*}\right]},\left\|I_{i}{ }^{\prime}-I_{i}{ }^{\prime \prime}\right\|_{L^{2}\left[(0, T),\left(W^{1,2}(\Omega)\right)^{*}\right]}^{2},\left\|I_{e}{ }^{\prime}-I_{e}{ }^{\prime \prime}\right\|_{L^{2}\left[(0, T),\left(W^{1,2}(\Omega)\right)^{*}\right]}^{2}\right) .
\end{aligned}
$$

- Step 7. Derivation of the estimate for $\left\|\Phi_{e}{ }^{\prime}-\Phi_{e}{ }^{\prime \prime}\right\|_{L^{2}\left[(0, T), W^{1,2}(\Omega)\right.}^{2}$ from the difference of the elliptic equations. Forming the difference of the equations

$$
\begin{aligned}
& \int_{\Omega}\left(\nabla \psi^{\mathrm{T}} M_{i} \nabla \Phi_{t r}{ }^{\prime}+\nabla \psi^{\mathrm{T}}\left(M_{i}+M_{e}\right) \nabla \Phi_{e}{ }^{\prime}\right) d x=\int_{\Omega}\left(I_{i}{ }^{\prime}+I_{e}{ }^{\prime}\right) \psi d x \text { and } \\
& \int_{\Omega}\left(\nabla \psi^{\mathrm{T}} M_{i} \nabla \Phi_{t r}^{\prime \prime}+\nabla \psi^{\mathrm{T}}\left(M_{i}+M_{e}\right) \nabla \Phi_{e}^{\prime \prime}\right) d x=\int_{\Omega}\left(I_{i}^{\prime \prime}+I_{e}{ }^{\prime \prime}\right) \psi d x
\end{aligned}
$$

$\forall \psi \in W^{1,2}(\Omega)$ with $\int_{\Omega} \psi(x) d x=0$,

which are valid for the pairs $\left(\Phi_{t r}{ }^{\prime}, \Phi_{e}{ }^{\prime}\right)$ and $\left(\Phi_{t r}{ }^{\prime \prime}, \Phi_{e}{ }^{\prime \prime}\right)$ for almost all $t \in[0, T]$, we get

$$
\begin{aligned}
\int_{\Omega}\left(\nabla \psi^{\mathrm{T}} M_{i} \nabla\left(\Phi_{t r}{ }^{\prime}-\Phi_{t r}{ }^{\prime \prime}\right)\right. & \left.+\nabla \psi^{\mathrm{T}}\left(M_{i}+M_{e}\right) \nabla\left(\Phi_{e}{ }^{\prime}-\Phi_{e}{ }^{\prime \prime}\right)\right) d x \\
= & \int_{\Omega}\left(\left(I_{i}{ }^{\prime}-I_{i}{ }^{\prime \prime}\right)+\left(I_{e}{ }^{\prime}-I_{e}{ }^{\prime \prime}\right) \psi d x \quad \forall \psi \in W^{1,2}(\Omega) \text { with } \int_{\Omega} \psi(x) d x=0 .\right.
\end{aligned}
$$

Inserting $\psi=\Phi_{e}{ }^{\prime}(t)-\Phi_{e}{ }^{\prime \prime}(t)$ as a feasible test function (cf. Definition 2.2.), we obtain from Assumption 2.3., 2) and the Poincaré inequality

$$
\begin{aligned}
& C\left\|\Phi_{e}{ }^{\prime}(t)-\Phi_{e}{ }^{\prime \prime}(t)\right\|_{W^{1,2}(\Omega)}^{2} \leqslant \int_{\Omega} \nabla\left(\Phi_{e}{ }^{\prime}-\Phi_{e}{ }^{\prime \prime}\right)^{\mathrm{T}} M_{i} \nabla\left(\Phi_{t r}{ }^{\prime}-\Phi_{t r}{ }^{\prime \prime}\right) d x \\
& +\int_{\Omega}\left(I_{i}{ }^{\prime}-I_{i}{ }^{\prime \prime}\right)\left(\Phi_{e}{ }^{\prime}-\Phi_{e}{ }^{\prime \prime}\right) d x+\int_{\Omega}\left(I_{e}{ }^{\prime}-I_{e}{ }^{\prime \prime}\right)\left(\Phi_{e}{ }^{\prime}-\Phi_{e}{ }^{\prime \prime}\right) d x \\
& \leqslant \frac{1}{2 \varepsilon_{5}}\left\|M_{i}\right\|^{2} \cdot\left\|\nabla \Phi_{t r}{ }^{\prime}-\nabla \Phi_{t r}{ }^{\prime \prime}\right\|_{L^{2}(\Omega)}^{2}+\frac{\varepsilon_{5}}{2}\left\|\nabla \Phi_{e}{ }^{\prime}-\nabla \Phi_{e}{ }^{\prime \prime}\right\|_{L^{2}(\Omega)}^{2}+\frac{1}{2 \varepsilon_{6}}\left\|I_{i}{ }^{\prime}(t)-I_{i}{ }^{\prime \prime}(t)\right\|^{2}\left(W^{1,2}(\Omega)\right)^{*} \\
& \left.+\frac{\varepsilon_{6}}{2}\left\|\Phi_{e}{ }^{\prime}-\Phi_{e}^{\prime \prime}\right\|_{W^{1,2}(\Omega)}^{2}+\frac{1}{2 \varepsilon_{7}}\left\|I_{e}{ }^{\prime}(t)-I_{e}{ }^{\prime \prime}(t)\right\|_{\left(W^{1,2}(\Omega)\right.}^{2}\right)^{*}+\frac{\varepsilon_{7}}{2}\left\|\Phi_{e}{ }^{\prime}-\Phi_{e}{ }^{\prime \prime}\right\|_{W^{1,2}(\Omega)}^{2} \\
& \leqslant \frac{C}{2 \varepsilon_{5}}\left\|\Phi_{t r}{ }^{\prime}-\Phi_{t r}{ }^{\prime \prime}\right\|_{W^{1,2}(\Omega)}^{2}+\frac{\varepsilon_{5}}{2}\left\|\Phi_{e}{ }^{\prime}-\Phi_{e}{ }^{\prime \prime}\right\|_{W^{1,2}(\Omega)}^{2}+\frac{1}{2 \varepsilon_{6}}\left\|I_{i}{ }^{\prime}(t)-I_{i}{ }^{\prime \prime}(t)\right\|_{\left(W^{1,2}(\Omega)\right)^{*}}^{2} \\
& \left.+\frac{\varepsilon_{6}}{2}\left\|\Phi_{e}{ }^{\prime}-\Phi_{e}{ }^{\prime \prime}\right\|_{W^{1,2}(\Omega)}^{2}+\frac{1}{2 \varepsilon_{7}}\left\|I_{e}{ }^{\prime}(t)-I_{e}{ }^{\prime \prime}(t)\right\|_{\left(W^{1,2}(\Omega)\right.}^{2}\right)^{*}+\frac{\varepsilon_{7}}{2}\left\|\Phi_{e}{ }^{\prime}-\Phi_{e}{ }^{\prime \prime}\right\|_{W^{1,2}(\Omega)}^{2} . \\
& \left\|\Phi_{e}{ }^{\prime}(t)-\Phi_{e}^{\prime \prime}(t)\right\|_{W^{1,2}(\Omega)}^{2} \leqslant C\left(\left\|\Phi_{t r}{ }^{\prime}(t)-\Phi_{t r}{ }^{\prime \prime}(t)\right\|_{W^{1,2}(\Omega)}^{2}\right. \\
& \left.+\left\|I_{i}{ }^{\prime}(t)-I_{i}{ }^{\prime \prime}(t)\right\|_{\left(W^{1,2}(\Omega)\right)^{*}}^{2}+\left\|I_{e}{ }^{\prime}(t)-I_{e}{ }^{\prime \prime}(t)\right\|_{\left(W^{1,2}(\Omega)\right)^{*}}^{2}\right) \Longrightarrow \\
& \left\|\Phi_{e}{ }^{\prime}-\Phi_{e}{ }^{\prime \prime}\right\|_{L^{2}\left[(0, T), W^{1,2}(\Omega)\right.}^{2}\left[\leqslant C\left(\left\|\Phi_{t r}{ }^{\prime}-\Phi_{t r}{ }^{\prime \prime}\right\|_{L^{2}\left[(0, T), W^{1,2}(\Omega)\right.}^{2}[\right.\right. \\
& \left.+\left\|I_{i}{ }^{\prime}-I_{i}{ }^{\prime \prime}\right\|_{L^{2}\left[(0, T),\left(W^{1,2}(\Omega)\right)^{*}\right]}^{2}+\left\|I_{e}{ }^{\prime}-I_{e}{ }^{\prime \prime}\right\|_{L^{2}\left[(0, T),\left(W^{1,2}(\Omega)\right)^{*}\right]}^{2}\right),
\end{aligned}
$$

from which we get with (2.87) the estimate

$$
\left\|\Phi_{e}{ }^{\prime}-\Phi_{e}{ }^{\prime \prime}\right\|_{W^{1,2}(\Omega)}^{2} \leqslant C\left(\left\|I_{i}{ }^{\prime}-I_{i}^{\prime \prime}\right\|_{L^{2}\left[(0, T),\left(W^{1,2}(\Omega)\right)^{*}\right]}^{2}+\left\|I_{e}{ }^{\prime}-I_{e}{ }^{\prime \prime}\right\|_{L^{2}\left[(0, T),\left(W^{1,2}(\Omega)\right)^{*}\right]}^{2}\right) .
$$


- Step 8. Conclusion of the proof. In order to confirm that the left-hand side in (2.81) can be replaced by $\left\|\Phi_{t r}{ }^{\prime}-\Phi_{t r}{ }^{\prime \prime}\right\|_{C^{0}\left[[0, T], L^{2}(\Omega)\right]}$, we may simply repeat the arguments from [BouRGault/Coudière/PierRE $09]$, p. 478, Subsection 5.3. The bound for $\left\|W^{\prime}-W^{\prime \prime}\right\|_{C^{0}\left[[0, T], L^{2}(\Omega)\right.}$ is obtained with [Kunisch/WAGNER 11], p. 21, Theorem 4.5., from (2.82) and (2.91). Finally, the estimates (2.38) and (2.39) take the claimed form due to the continous imbedding $L^{\infty}\left[(0, T),\left(W^{1,2}(\Omega)\right)^{*}\right] \hookrightarrow L^{2}\left[(0, T),\left(W^{1,2}(\Omega)\right)^{*}\right]$, and the proof is complete.

Remarks. 1) Considering the system (B) $)_{2}$ with the FitzHugh-Nagumo instead of the Rogers-McCulloch model, thus replacing the nonlinear coupling term $\varphi w$ by $w$ and setting $b=1$ within the ionic current, the preceding proof can be repeated with almost no alterations. The only difference concerns the estimation of the term

$$
\int_{\Omega}\left(I_{i o n}\left(\Phi_{t r}^{\prime}, W^{\prime}\right)-I_{i o n}\left(\Phi_{t r}^{\prime \prime}, W^{\prime \prime}\right)\right)\left(\Phi_{t r}^{\prime}-\Phi_{t r}^{\prime \prime}\right) d x
$$

within $(2.57)$.

2) Assume now that in $(\mathrm{B})_{2}$ the linearized Aliev-Panfilov model is specified. Then in the proof above, the estimates for the gating equation in Steps 2 and 5 must be changed. Namely, the difference of the gating equations for two weak solutions $\left(\Phi_{t r}{ }^{\prime}, \Phi_{e}{ }^{\prime}, W^{\prime}\right)$ and $\left(\Phi_{t r}{ }^{\prime \prime}, \Phi_{e}{ }^{\prime \prime}, W^{\prime \prime}\right)$ turns into

$$
\begin{aligned}
& \left\langle\frac{d}{d t}\left(W^{\prime}(t)-W^{\prime \prime}(t)\right), \psi\right\rangle=-\varepsilon \int_{\Omega}\left(W^{\prime}(t)-W^{\prime \prime}(t)\right) \psi d x \\
& +\varepsilon \kappa(a+1) \int_{\Omega}\left(\Phi_{t r}{ }^{\prime}(t)-\Phi_{t r}{ }^{\prime \prime}(t)\right) \psi d x-\varepsilon \kappa \int_{\Omega}\left(\Phi_{t r}{ }^{\prime}(t)^{2}-\Phi_{t r}{ }^{\prime \prime}(t)^{2}\right) \psi d x \quad \forall \psi \in L^{2}(\Omega) .
\end{aligned}
$$

Inserting now the feasible test function $\psi=W^{\prime}(t)-W^{\prime \prime}(t)$, we obtain

$$
\begin{aligned}
& \left\langle\frac{d}{d t}\left(W^{\prime}(t)-W^{\prime \prime}(t)\right), W^{\prime}(t)-W^{\prime \prime}(t)\right\rangle=-\varepsilon \int_{\Omega}\left(W^{\prime}-W^{\prime \prime}\right)^{2} d x \\
& +\varepsilon \kappa(a+1) \int_{\Omega}\left(\Phi_{t r}{ }^{\prime}-\Phi_{t r}{ }^{\prime \prime}\right)\left(W^{\prime}-W^{\prime \prime}\right) d x-\varepsilon \kappa \int_{\Omega}\left(\left(\Phi_{t r}{ }^{\prime}\right)^{2}-\left(\Phi_{t r}{ }^{\prime \prime}\right)^{2}\right)\left(W^{\prime}-W^{\prime \prime}\right) d x \Longrightarrow \\
& \frac{d}{d t}\left(\frac{1}{2}\left\|W^{\prime}-W^{\prime \prime}\right\|_{L^{2}(\Omega)}^{2}\right) \leqslant \varepsilon\left\|W^{\prime}-W^{\prime \prime}\right\|_{L^{2}(\Omega)}^{2}+\varepsilon \kappa(a+1) \int_{\Omega}\left|\Phi_{t r}{ }^{\prime}-\Phi_{t r}{ }^{\prime \prime}\right| \cdot\left|W^{\prime}-W^{\prime \prime}\right| d x \\
& +\varepsilon \kappa \int_{\Omega}\left|\left(\Phi_{t r}{ }^{\prime}\right)^{2}-\left(\Phi_{t r}{ }^{\prime \prime}\right)^{2}\right| \cdot\left|W^{\prime}-W^{\prime \prime}\right| d x \Longrightarrow \\
& \frac{d}{d t}\left(\left\|W^{\prime}-W^{\prime \prime}\right\|_{L^{2}(\Omega)}^{2}\right) \leqslant(2 \varepsilon+\varepsilon \kappa(a+1))\left\|W^{\prime}-W^{\prime \prime}\right\|_{L^{2}(\Omega)}^{2}+\varepsilon \kappa(a+1)\left\|\Phi_{t r}{ }^{\prime}-\Phi_{t r}{ }^{\prime \prime}\right\|_{L^{2}(\Omega)}^{2} \\
& +2 \varepsilon_{8} \int_{\Omega}\left(\Phi_{t r}{ }^{\prime}+\Phi_{t r}{ }^{\prime \prime}\right)^{2}\left(\Phi_{t r}{ }^{\prime}-\Phi_{t r}{ }^{\prime \prime}\right)^{2} d x+\frac{1}{2 \varepsilon_{8}} \int_{\Omega}\left(W^{\prime}-W^{\prime \prime}\right)^{2} d x \\
& \leqslant C\left(\left\|\Phi_{t r}^{\prime}-\Phi_{t r}^{\prime \prime}\right\|_{L^{2}(\Omega)}^{2}+\left\|W^{\prime}-W^{\prime \prime}\right\|_{L^{2}(\Omega)}^{2}\right) \\
& +C \varepsilon_{8}\left(\int_{\Omega}\left(\left|\Phi_{t r}{ }^{\prime}\right|^{4}+\left|\Phi_{t r}{ }^{\prime \prime}\right|^{4}\right) d x\right)^{1 / 2}\left\|\Phi_{t r}{ }^{\prime}-\Phi_{t r}{ }^{\prime \prime}\right\|_{L^{4}(\Omega)}^{2}+\frac{1}{2 \varepsilon_{8}}\left\|W^{\prime}-W^{\prime \prime}\right\|_{L^{2}(\Omega)}^{2}
\end{aligned}
$$

where the generalized Cauchy's inequality with $\varepsilon_{8}>0$ has been applied. Together with (2.69), (2.70) and the imbedding $W^{1,2}(\Omega) \hookrightarrow L^{4}(\Omega)$, we find

$\frac{d}{d t}\left(\left\|W^{\prime}-W^{\prime \prime}\right\|_{L^{2}(\Omega)}^{2}\right) \leqslant\left(C+\frac{1}{2 \varepsilon_{8}}\right)\left(\left\|\Phi_{t r}{ }^{\prime}-\Phi_{t r}^{\prime \prime}\right\|_{L^{2}(\Omega)}^{2}+\left\|W^{\prime}-W^{\prime \prime}\right\|_{L^{2}(\Omega)}^{2}\right)$

$$
+C \varepsilon_{8}\left\|\Phi_{t r}{ }^{\prime}-\Phi_{t r}{ }^{\prime \prime}\right\|_{W^{1,2}(\Omega)}^{2}
$$


as a replacement of (2.77). In Step 5 above, the corresponding alterations will be applied.

Proof of Theorem 2.8. For any of the three ionic models, the existence of a weak solution $\left(\Phi_{t r}, \Phi_{e}, W\right)$ of $(B)_{2}$ is ensured by Theorem 2.5. In order to prove its uniqueness, Theorem 2.7. has to be applied to $I_{i}{ }^{\prime}=I_{i}{ }^{\prime \prime}=I_{i}$ and $I_{e}{ }^{\prime}=I_{e}{ }^{\prime \prime}=I_{e}$.

\section{Higher regularity of weak solutions for the bidomain system.}

\section{a) Veneroni's iterative procedure.}

In order to prove a further regularity theorem for the weak solutions of $(B)_{2}$, we strengthen the assumptions on the data. In particular, we will assume throughout this section that the sum of the excitations $I_{i}$ and $I_{e}$ possesses a weak time derivative.

Assumptions 3.1. (Stronger assumptions on the data in $(\mathrm{B})_{1}$ and $(\mathrm{B})_{2}$ )

1) $\Omega \subset \mathbb{R}^{3}$ is a bounded domain with $C^{1,1}$-boundary.

2) $M_{i}, M_{e}: \operatorname{cl}(\Omega) \rightarrow \mathbb{R}^{3 \times 3}$ are symmetric, positive definite matrix functions with $W^{1, \infty}(\Omega)$-coefficients, obeying uniform ellipticity conditions:

$0 \leqslant \mu_{1}\|\xi\|^{2} \leqslant \xi^{\mathrm{T}} M_{i}(x) \xi \leqslant \mu_{2}\|\xi\|^{2}$ and $0 \leqslant \mu_{1}\|\xi\|^{2} \leqslant \xi^{\mathrm{T}} M_{e}(x) \xi \leqslant \mu_{2}\|\xi\|^{2} \quad \forall \xi \in \mathbb{R}^{3} \quad \forall x \in \Omega$

with $\mu_{1}, \mu_{2}>0$.

3) The initial values belong to the following spaces: $\Phi_{0} \in W^{2,2}(\Omega), W_{0} \in L^{\infty}(\Omega)$. Moreover, $\Phi_{0}$ satisfies the compatibility condition described in [VENERONI 09], p. 853, and p. 854, Remark 3. ${ }^{27)}$

4) $I_{i}, I_{e}$ belong to the space $L^{r}\left[(0, T), L^{2}(\Omega)\right]$ with $r>4$; the sum $I_{i}+I_{e}$ belongs even to the Sobolev space $W^{1,2}\left[(0, T), L^{2}(\Omega)\right]$, and it holds that

$$
\int_{\Omega}\left(I_{i}(x, t)+I_{e}(x, t)\right) d x=0 \quad(\forall) t \in(0, \infty)
$$

Note that Assumption 3.1., $k$ ) implies Assumption 2.3., $k$ ) from Subsection 2.a), $1 \leqslant k \leqslant 4$.

The following theorem is part of an iterative solution procedure for the bidomain system, which has been presented in [VENERONI 09]. In the present investigation, Veneroni's result will be used in order to improve the regularity of a given weak solution of $(\mathrm{B})_{2}$.

Theorem 3.2. (Iterative solution of the bidomain system) ${ }^{28)}$ Let Assumptions 3.1. hold. Further, let a function $\widetilde{\Phi}_{t r} \in L^{2}\left[(0, T), W^{1,2}(\Omega)\right]$ be given, which is regular enough to ensure that $I_{i o n}\left(\widetilde{\Phi}_{t r}, W\left(\widetilde{\Phi}_{t r}\right)\right)$ belongs to $L^{q}\left[(0, T), L^{2}(\Omega)\right], q>1$, where $W\left(\widetilde{\Phi}_{t r}\right) \in C^{0}\left[[0, T], L^{2}(\Omega)\right]$ is defined as the (weak or strong) solution of the initial value problem

$(\widetilde{\mathrm{B}})_{0, g a t} \quad \frac{\partial W}{\partial t}+G\left(\widetilde{\Phi}_{t r}, W\right)=0(\forall)(x, t) \in \Omega \times(0, T), \quad W(x, 0)=W_{0}(x)(\forall) x \in \Omega$.

27) In contrast to the condition from [Bourgault/Coudière/Pierre 09], p. 469, Theorem 20, this condition can be verified in a constructive way.

28) [Veneroni 09] , p. 856 f., Proposition 3.1., together with p. 861. 
1) Then the initial-boundary value problem

$$
\begin{aligned}
(\widetilde{\mathrm{B}})_{0, p a r} \quad & \frac{\partial \Phi_{t r}}{\partial t}+I_{i o n}\left(\widetilde{\Phi}_{t r}, W\left(\widetilde{\Phi}_{t r}\right)\right)-\operatorname{div}\left(M_{i} \nabla \Phi_{i}\right)=\quad I_{i} \quad(\forall)(x, t) \in \Omega \times[0, T] \\
& \frac{\partial \Phi_{t r}}{\partial t}+I_{i o n}\left(\widetilde{\Phi}_{t r}, W\left(\widetilde{\Phi}_{t r}\right)\right)+\operatorname{div}\left(M_{e} \nabla \Phi_{e}\right)=-I_{e} \quad(\forall)(x, t) \in \Omega \times[0, T] \\
& \mathfrak{n}^{\mathrm{T}} M_{i} \nabla \Phi_{i}=0 \quad \forall(x, t) \in \partial \Omega \times[0, T] ; \\
& \mathfrak{n}^{\mathrm{T}} M_{e} \nabla \Phi_{e}=0 \quad \forall(x, t) \in \partial \Omega \times[0, T] ; \\
& \Phi_{t r}(x, 0)=\Phi_{i}(x, 0)-\Phi_{e}(x, 0)=\Phi_{0}(x) \quad(\forall) x \in \Omega ; \quad \int_{\Omega} \Phi_{e}(x, t) d x=0 \quad(\forall) t \in[0, T]
\end{aligned}
$$

possesses a uniquely determined solution $\left(\Phi_{i}, \Phi_{e}\right) \in L^{q}\left[(0, T), W^{2,2}(\Omega)\right] \times L^{q}\left[(0, T), W^{2,2}(\Omega)\right]$ with

$$
\Phi_{t r}=\Phi_{i}-\Phi_{e} \in W^{1, q}\left[(0, T), L^{2}(\Omega)\right] \cap L^{q}\left[(0, T), W^{2,2}(\Omega)\right]
$$

2) The solutions $\Phi_{i}, \Phi_{e}$ satisfy the estimates

$$
\begin{aligned}
& \left\|\Phi_{i}\right\|_{L^{q}\left[(0, T), W^{2,2}(\Omega)\right]}+\left\|\Phi_{t r}\right\|_{W^{1, q}\left[(0, T), L^{2}(\Omega)\right]} \leqslant C\left(\left\|\Phi_{0}\right\|_{W^{2,2}(\Omega)}\right. \\
& \left.\quad+\left\|I_{i o n}\left(\widetilde{\Phi}_{t r}, W\left(\widetilde{\Phi}_{t r}\right)\right)\right\|_{L^{q}\left[(0, T), L^{2}(\Omega)\right]}+\left\|I_{i}\right\|_{L^{q}\left[(0, T), L^{2}(\Omega)\right]}+\left\|I_{i}+I_{e}\right\|_{W^{1,2}\left[(0, T), L^{2}(\Omega)\right]}\right) \\
& \left\|\Phi_{e}\right\|_{L^{q}\left[(0, T), W^{2,2}(\Omega)\right]}+\left\|\Phi_{t r}\right\|_{W^{1, q}\left[(0, T), L^{2}(\Omega)\right]} \leqslant C\left(\left\|\Phi_{0}\right\|_{W^{2,2}(\Omega)}\right. \\
& \left.\quad+\left\|I_{i o n}\left(\widetilde{\Phi}_{t r}, W\left(\widetilde{\Phi}_{t r}\right)\right)\right\|_{L^{q}\left[(0, T), L^{2}(\Omega)\right]}+\left\|I_{e}\right\|_{L^{q}\left[(0, T), L^{2}(\Omega)\right]}+\left\|I_{i}+I_{e}\right\|_{W^{1,2}\left[(0, T), L^{2}(\Omega)\right]}\right)
\end{aligned}
$$

3) If $q \geqslant 2$ then for a pair of solutions $\left(\Phi_{t r}{ }^{\prime}, \Phi_{e}{ }^{\prime}, W^{\prime}\right),\left(\Phi_{t r}{ }^{\prime \prime}, \Phi_{e}{ }^{\prime \prime}, W^{\prime \prime}\right)$ of $(\widetilde{\mathrm{B}})_{0, \text { gat }}$ and $(\widetilde{\mathrm{B}})_{0, \text { par }}$, corresponding to the entries $\left(\widetilde{\Phi}_{t r}^{\prime}, \widetilde{\Phi}_{e}^{\prime}, \widetilde{W}^{\prime}\right)$ and $\left(\widetilde{\Phi}_{t r}^{\prime \prime}, \widetilde{\Phi}_{e}^{\prime \prime}, \widetilde{W}^{\prime \prime}\right)$, we have the estimate

$$
\left\|\Phi_{t r}^{\prime}(t)-\Phi_{t r}^{\prime \prime}(t)\right\|_{L^{2}(\Omega)}^{2} \leqslant C \int_{0}^{t}\left\|I_{i o n}\left(\widetilde{\Phi}_{t r}^{\prime}(\vartheta), \widetilde{W}^{\prime}(\vartheta)\right)-I_{i o n}\left(\widetilde{\Phi}_{t r}^{\prime \prime}(\vartheta), \widetilde{W}^{\prime \prime}(\vartheta)\right)\right\|_{L^{2}(\Omega)}^{2} d \vartheta \forall t \in[0, T]
$$

Proof. Parts 1) and 2) of Theorem 3.1. are identical with [Veneroni 09], p. 856 f., Proposition 3.1., except for the fact that $I_{i o n}\left(\widetilde{\Phi}_{t r}, W\left(\widetilde{\Phi}_{t r}\right)\right)$ is assumed to belong to $L^{q}\left[(0, T), L^{2}(\Omega)\right], 1<q \leqslant r$, instead of $L^{r}\left[(0, T), L^{2}(\Omega)\right]$. However, a closer inspection of the proof in [VENERONI 09], pp. 858-861, reveals that all arguments remain in virtue for the above assumed weaker regularity since the $W^{1, q}$-regularity of the solution of $(\widetilde{\mathrm{B}})_{0, p a r}$ depends on the regularity of

$$
\frac{I_{i}(t)+I_{e}(t)}{2}-I_{i o n}\left(\widetilde{\Phi}_{t r}(t), W\left(\widetilde{\Phi}_{t r}(t)\right)\right) \in L^{q}(\Omega)
$$

only. Then the estimates in Part 2) and 3) follow from [Veneroni 09], p. 861, Theorem 3.1., together with p. $860,(3.27),(3.28)$ and $(3.30)$, in a completely analogous way.

\section{b) Higher regularity of weak solutions.}

Imposing the Assumptions 3.1., the regularity of a given weak solution of $(\mathrm{B})_{2}$ can be considerably improved.

Theorem 3.3. (Higher regularity of weak solutions) Let the Assumptions 3.1. with $r>4$ hold, and specify within $(\mathrm{B})_{2}$ one of the models from Subsection 2.b). Assume that a triple $\left(\Phi_{t r}, \Phi_{e}, W\right)$ forms a weak 
solution of the bidomain system $(\mathrm{B})_{2}$ on $[0, T]$ in correspondence to initial values $\Phi_{0} \in W^{2,2}(\Omega)$ and $W_{0} \in L^{\infty}(\Omega)$ where $p=4$. Then the functions possess the higher regularity

$$
\begin{aligned}
& \Phi_{t r} \in W^{1,6}\left[(0, T), L^{2}(\Omega)\right] \cap L^{6}\left[(0, T), W^{2,2}(\Omega)\right] \cap C^{0}\left(\Omega_{T}\right) ; \\
& \Phi_{e} \in L^{2}\left[(0, T), L^{2}(\Omega)\right] \cap L^{6}\left[(0, T), W^{2,2}(\Omega)\right] ; \\
& W \in C^{1}\left[(0, T), L^{\infty}(\Omega)\right] \cap C^{0}\left[[0, T], L^{\infty}(\Omega)\right] .
\end{aligned}
$$

Consequently, $\left(\Phi_{t r}, \Phi_{e}, W\right)$ forms even a strong solution of $(\mathrm{B})_{0}$ and $(\mathrm{B})_{1}$.

Corollary 3.4. (Higher regularity and uniqueness of weak solutions) Let the assumptions of Theorem 3.3. hold. Besides Assumption 3.1., 4), let the inhomogeneities $I_{i}$ and $I_{e}$ belong even to the space $L^{\infty}\left[(0, T), L^{2}(\Omega)\right]$. Then the bidomain system $(\mathrm{B})_{2}$ possesses on $[0, T]$ a uniquely determined weak solution $\left(\Phi_{t r}, \Phi_{e}, W\right)$, which admits the regularity described by (3.14) - (3.16) and, consequently, forms a uniquely determined strong solution of $(\mathrm{B})_{0}$ and $(\mathrm{B})_{1}$ as well.

As pointed out e. g. in [Colli Franzone/Guerri/Tentoni 90], p. 159 f., the eigenvector bases of the conductivity tensors $M_{i}, M_{e}: \operatorname{cl}(\Omega) \rightarrow \mathbb{R}^{3 \times 3}$, reflecting the direction of the muscle fibers within the heart tissue, should be identical. Consequently, let us assume that the representations

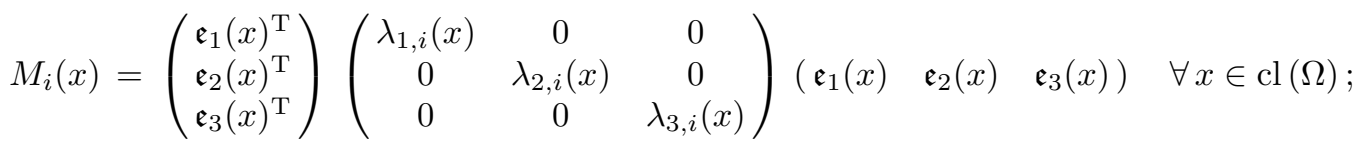

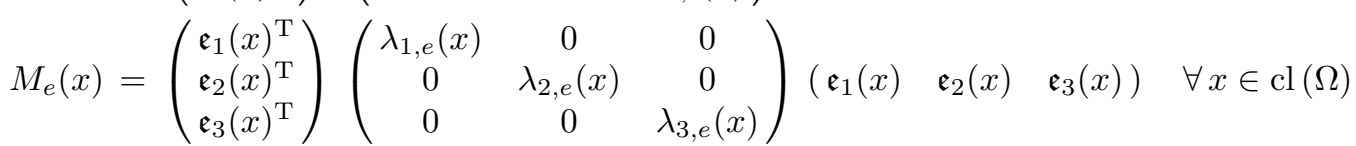

hold. As a consequence, the boundary conditions as well as the compatibility condition for the initial datum $\Phi_{0}$ may be simplified in the following way:

Corollary 3.5. (Geometrical consequences of higher regularity) Let the assumptions of Theorem 3.3. hold. Assume further that the descriptions (3.17), (3.18) are valid together with

$M_{i}(x) \mathfrak{n}(x)=\left(\begin{array}{ccc}\lambda_{1, i}(x) & 0 & 0 \\ 0 & \lambda_{2, i}(x) & 0 \\ 0 & 0 & \lambda_{3, i}(x)\end{array}\right) \mathfrak{n}(x), M_{e}(x) \mathfrak{n}(x)=\left(\begin{array}{ccc}\lambda_{1, e}(x) & 0 & 0 \\ 0 & \lambda_{2, e}(x) & 0 \\ 0 & 0 & \lambda_{3, e}(x)\end{array}\right) \mathfrak{n}(x)$

$\forall x \in \partial \Omega$

while $\mathfrak{e}_{1}, \mathfrak{e}_{2}, \mathfrak{e}_{3}: \operatorname{cl}(\Omega) \rightarrow \mathbb{R}^{3}$ are continuous functions.

1) Then any weak solution of $(\mathrm{B})_{2}$ on $[0, T]$ is a strong solution of $(\mathrm{B})_{0}$ and $(\mathrm{B})_{1}$ where the boundary conditions $(1.4)-(1.5)$ and $(2.4)-(2.5)$ can be replaced by

$$
\begin{array}{lll}
\mathfrak{n}^{\mathrm{T}} \nabla \Phi_{i}=0, & \mathfrak{n}^{\mathrm{T}} \nabla \Phi_{e}=0 & \forall(x, t) \in \partial \Omega \times[0, T] \quad \text { and } \\
\mathfrak{n}^{\mathrm{T}} \nabla \Phi_{t r}=0, & \mathfrak{n}^{\mathrm{T}} \nabla \Phi_{e}=0 & \forall(x, t) \in \partial \Omega \times[0, T],
\end{array}
$$

respectively.

2) The compatibility condition for the function $\Phi_{0} \in W^{2,2}(\Omega)$ from Assumption 3.1., 3) reduces to

$$
\mathfrak{n}^{\mathrm{T}} \nabla \Phi_{0}=0 \quad \forall x \in \partial \Omega .
$$

The proofs of Theorem 3.3. and the Corollaries 3.4. and 3.5. will be given in the following subsection. 
Remark. By a slight modification of the full iteration procedure from [VENERONI 09], the uniqueness of the weak solutions of $(B)_{2}$ can be confirmed exclusively under the assumptions of Theorem 3.3. without reference to Theorem 2.8. In the optimal control problems for the bidomain system, however, the intracellular excitation $I_{i}$ is set zero for physiological reasons; consequently, Assumption 3.1., 4) implies $I_{e} \in W^{1,2}\left[(0, T), L^{2}(\Omega)\right]$, which is continuously imbedded into $C^{0}\left[[0, T], L^{2}(\Omega)\right] \subset L^{\infty}\left[(0, T), L^{2}(\Omega)\right]$ by [KUnisch/WAGNER 11 ], p. 21, Theorem 4.5, and we arrive at the analytical situation of Corollary 3.4. from the outset.

\section{c) Proofs.}

Before starting with the proof of Theorem 3.3., we establish the following two propositions.

Proposition 3.6. (Iterative definition of the entries for the bidomain system I) Under the assumptions of Theorem 3.3., let a triple $\left(\widetilde{\Phi}_{t r}, \widetilde{\Phi}_{e}, \widetilde{W}\right) \in\left(C^{0}\left[[0, T], L^{2}(\Omega)\right] \cap L^{2}\left[(0, T), W^{1,2}(\Omega)\right] \cap\right.$ $\left.L^{4}\left(\Omega_{T}\right)\right) \times L^{2}\left[(0, T), W^{1,2}(\Omega)\right] \times C^{0}\left[[0, T], L^{2}(\Omega)\right]$ be a weak solution of the bidomain system $(\mathrm{B})_{2}$ on $[0, T]$.

1) In the case of the Rogers-McCulloch and the FitzHugh-Nagumo model, the (weak or strong) solution $W$ of the initial value problem $(\widetilde{\mathrm{B}})_{0, \text { gat }}$ from Theorem 3.2. belongs to the space $C^{1}\left[(0, T), L^{2}(\Omega)\right] \cap$ $C^{0}\left[[0, T], L^{2}(\Omega)\right]$ and coincides with $\widetilde{W}$.

2) In the case of the linearized Aliev-Panfilov model, the (weak or strong) solution $W$ of the initial value problem $(\widetilde{\mathrm{B}})_{0, \text { gat }}$ from Theorem 3.2. belongs to the space $C^{1}\left[(0, T), L^{1}(\Omega)\right] \cap C^{0}\left[[0, T], L^{2}(\Omega)\right]$ and coincides with $\widetilde{W}$.

Proof. 1) In the case of the Rogers-McCulloch model and the FitzHugh-Nagumo model, the (weak or strong) solution of $(\widetilde{\mathrm{B}})_{0, \text { gat }}$ is given through

$$
W(x, t)=W_{0}(x) e^{-\varepsilon t}+\varepsilon \kappa e^{-\varepsilon t} \int_{0}^{t} \widetilde{\Phi}_{t r}(x, \tau) e^{\varepsilon \tau} d \tau,
$$

cf. [WARGA 72] , p. 192, Theorem II.4.6., and (2.71) above. Obviously, this function admits the claimed regularity. Since (3.23) is the uniquely determined weak solution of $(\widetilde{\mathrm{B}})_{0, \text { gat }}$ as well, the solution $W$ of $(\widetilde{\mathrm{B}})_{0, \text { gat }}$ must agree with $\widetilde{W}$, which is already known as a weak solution of $(\widetilde{\mathrm{B}})_{0, \text { gat }}$. Consequently, $W$ and $\widetilde{W}$ agree even within the space $C^{1}\left[(0, T), L^{2}(\Omega)\right] \cap C^{0}\left[[0, T], L^{2}(\Omega)\right]$.

2) In the case of the linearized Aliev-Panfilov model, the (weak or strong) solution of $(\widetilde{\mathrm{B}})_{0, \text { gat }}$ reads as

$$
W(x, t)=W_{0}(x) e^{-\varepsilon t}+\varepsilon \kappa e^{-\varepsilon t} \int_{0}^{t}\left((a+1) \widetilde{\Phi}_{t r}(x, \tau)-\widetilde{\Phi}_{t r}^{2}(x, \tau)\right) e^{\varepsilon \tau} d \tau,
$$

and the same implications are true except for the lower integrability of $\partial W(x, t) / \partial t$ with respect to $x$.

Proposition 3.7. (Iterative definition of the entries for the bidomain system II) Let the assumptions of Theorem 3.3. hold. Consider a triple $\left(\widetilde{\Phi}_{t r}, \widetilde{\Phi}_{e}, \widetilde{W}\right) \in\left(C^{0}\left[[0, T], L^{2}(\Omega)\right] \cap L^{2}\left[(0, T), W^{1,2}(\Omega)\right] \cap\right.$ $\left.L^{4}\left(\Omega_{T}\right)\right) \times L^{2}\left[(0, T), W^{1,2}(\Omega)\right] \times C^{0}\left[[0, T), L^{2}(\Omega)\right]$, which forms on $[0, T]$ a weak solution of the bidomain system $(\mathrm{B})_{2}$ with initial values $\Phi_{0} \in W^{2,2}(\Omega)$ and $W_{0} \in L^{\infty}(\Omega)$. Assume further that $\widetilde{\Phi}_{\text {tr }}$ is regular enough to ensure that $I_{i o n}\left(\widetilde{\Phi}_{t r}, W\left(\widetilde{\Phi}_{t r}\right)\right)$ belongs to $L^{q}\left[(0, T), L^{2}(\Omega)\right], q>1$. Then the solutions $\Phi_{t r}$, $\Phi_{e}$ of $(\widetilde{\mathrm{B}})_{0, p a r}$ coincide with $\widetilde{\Phi}_{t r}$ and $\widetilde{\Phi}_{e}$ within the space $L^{2}\left[(0, T), W^{1,2}(\Omega)\right]$.

Proof. The assumptions about $\widetilde{\Phi}_{t r}$ guarantee the applicability of Theorem 3.2., 1). Consequently, the system $(\widetilde{\mathrm{B}})_{0, \text { par }}$ admits the uniquely determined solutions

$$
\begin{aligned}
& \Phi_{i}, \Phi_{e} \in L^{q}\left[(0, T), W^{2,2}(\Omega)\right] \text { with } \\
& \Phi_{t r}=\Phi_{i}-\Phi_{e} \in W^{1, q}\left[(0, T), L^{2}(\Omega)\right] \cap L^{q}\left[(0, T), W^{2,2}(\Omega)\right] .
\end{aligned}
$$


Due to Proposition 3.6., we have $W\left(\widetilde{\Phi}_{t r}\right)=\widetilde{W}$, and the difference of $(1.1)-(1.2),(1.4)-(1.6)$ and $(3.4)-$ (3.8) reads as

$$
\begin{aligned}
& \frac{\partial}{\partial t}\left(\widetilde{\Phi}_{t r}-\Phi_{t r}\right)-\operatorname{div}\left(M_{i} \nabla\left(\widetilde{\Phi}_{i}-\Phi_{i}\right)\right)=0 \quad(\forall)(x, t) \in \Omega \times[0, T] ; \\
& \frac{\partial}{\partial t}\left(\widetilde{\Phi}_{t r}-\Phi_{t r}\right)+\operatorname{div}\left(M_{e} \nabla\left(\widetilde{\Phi}_{e}-\Phi_{e}\right)\right)=0 \quad(\forall)(x, t) \in \Omega \times[0, T] ; \\
& \mathfrak{n}^{\mathrm{T}} M_{i} \nabla\left(\widetilde{\Phi}_{i}-\Phi_{i}\right)=0 \quad \text { and } \mathfrak{n}^{\mathrm{T}} M_{e} \nabla\left(\widetilde{\Phi}_{e}-\Phi_{e}\right)=0 \forall(x, t) \in \partial \Omega \times[0, T] ; \\
& \widetilde{\Phi}_{t r}(x, 0)-\Phi_{t r}(x, 0)=0 \quad(\forall) x \in \Omega ; \quad \int_{\Omega}\left(\widetilde{\Phi}_{e}(x, t)-\Phi_{e}(x, t)\right) d x=0 \quad(\forall) t \in[0, T] .
\end{aligned}
$$

Obviously, this system matches the assumptions of Theorem 3.2., 1) with $I_{\text {ion }}(\varphi, w)=\mathfrak{o}$; consequently, the zero solution $\left(\widetilde{\Phi}_{i}-\Phi_{i}\right)=\left(\widetilde{\Phi}_{e}-\Phi_{e}\right)=\mathfrak{o} \in L^{2}\left[(0, T), W^{1,2}(\Omega)\right]$ is uniquely determined, and the functions $\widetilde{\Phi}_{t r}$ and $\Phi_{t r}$ as well as $\widetilde{\Phi}_{e}$ and $\Phi_{e}$ are identical.

Proof of Theorem 3.3. Throughout the proof, $C$ denotes a generical positive constant, which may appropriately change from line to line. $C$ will never depend on the data $\Phi_{0}, W_{0}, I_{i}$ and $I_{e}$ but possibly on $\Omega$ and $p=4$. Within $(\mathrm{B})_{0},(\mathrm{~B})_{1}$ and $(\mathrm{B})_{2}$, the Rogers-McCulloch model is specified. In order to apply Theorem 3.2. and Propositions 3.6. and 3.7., we rename the given weak solution as $\left(\widetilde{\Phi}_{t r}, \widetilde{\Phi}_{e}, \widetilde{W}\right)$ and study the outcomes $W=W\left(\widetilde{\Phi}_{t r}\right)$ and $\left(\Phi_{t r}, \Phi_{e}\right)$ of Veneroni's procedure.

- Step 1. The function $\widetilde{\Phi}_{t r}$ belongs even to the space $L^{4}\left[(0, T), L^{6}(\Omega)\right]$. We start with the following estimate, which is taken from the proof of Theorem 2.6. in [NAGAIAH/Kunisch/Plank 11], p. 158, (33):

$$
\begin{aligned}
\left\|\widetilde{\Phi}_{t r}(t)\right\|_{W^{1,2}(\Omega)}^{2} \leqslant & C\left(1+\left\|\Phi_{0}\right\|_{L^{2}(\Omega)}^{2}+\left\|W_{0}\right\|_{L^{2}(\Omega)}^{2}\right. \\
& \left.+\left\|I_{i}(t)\right\|_{L^{2}(\Omega)}^{2}+\left\|I_{e}(t)\right\|_{L^{2}(\Omega)}^{2}+\int_{0}^{t}\left\|I_{i}(\tau)\right\|_{\left(W^{1,2}(\Omega)\right)}^{2} d \tau+\int_{0}^{t}\left\|I_{e}(\tau)\right\|_{\left(W^{1,2}(\Omega)\right)^{*}}^{2} d \tau\right) .
\end{aligned}
$$

Due to Assumption 3.1., 4), the following may be derived from (3.31):

$$
\begin{aligned}
& \left\|\widetilde{\Phi}_{t r}(t)\right\|_{W^{1,2}(\Omega)}^{4} \leqslant C\left(1+\left\|\Phi_{0}\right\|_{L^{2}(\Omega)}^{4}+\left\|W_{0}\right\|_{L^{2}(\Omega)}^{4}+\left\|I_{i}(t)\right\|_{L^{2}(\Omega)}^{4}+\left\|I_{e}(t)\right\|_{L^{2}(\Omega)}^{4}\right. \\
& \left.+\left\|I_{i}\right\|_{L^{2}\left[(0, T),\left(W^{1,2}(\Omega)\right)^{*}\right]}^{4}+\left\|I_{e}\right\|_{L^{2}\left[(0, T),\left(W^{1,2}(\Omega)\right)^{*}\right]}^{4}\right) \Longrightarrow \\
& \left.\left\|\widetilde{\Phi}_{t r}\right\|_{L^{4}\left[(0, T), W^{1,2}(\Omega)\right.}^{4}\right] \leqslant C T\left(1+\left\|\Phi_{0}\right\|_{L^{2}(\Omega)}^{4}+\left\|W_{0}\right\|_{L^{2}(\Omega)}^{4}+\left\|I_{i}\right\|_{L^{4}\left[(0, T), L^{2}(\Omega)\right]}^{4}\right. \\
& \left.+\left\|I_{e}\right\|_{L^{4}\left[(0, T), L^{2}(\Omega)\right.}^{4}+\left\|I_{i}\right\|_{L^{2}\left[(0, T),\left(W^{1,2}(\Omega)\right)^{*}\right]}^{4}+\left\|I_{e}\right\|_{L^{2}\left[(0, T),\left(W^{1,2}(\Omega)\right)^{*}\right]}^{4}\right) .
\end{aligned}
$$

Since the imbedding $W^{1,2}(\Omega) \hookrightarrow L^{6}(\Omega)$ is continuous, $\widetilde{\Phi}_{t r}$ is contained in the space $L^{4}\left[(0, T), L^{6}(\Omega)\right]$.

- Step 2. The function $I_{i o n}\left(\widetilde{\Phi}_{t r}, W\right)=I_{i o n}\left(\widetilde{\Phi}_{t r}, \widetilde{W}\right)$ belongs to the space $L^{4 / 3}\left[(0, T), L^{2}(\Omega)\right] \cdot{ }^{29)} \mathrm{By}(2.28)$, we may write

29) Note that, from [Bourgault/Coudière/Pierre 09], p. 471, Lemma 25, we only know that $I_{i o n}(\widetilde{\Phi}$ tr $(t), \widetilde{W}(t))$ belongs to $L^{4 / 3}(\Omega)$ for almost all $t \in(0, T)$. 


$$
\begin{aligned}
& \left\|I_{i o n}\left(\widetilde{\Phi}_{t r}(t), \widetilde{W}(t)\right)\right\|_{L^{2}(\Omega)}^{2}=\int_{\Omega}\left(I_{i o n}\left(\widetilde{\Phi}_{t r}(t), \widetilde{W}(t)\right)\right)^{2} d x \\
& =\int_{\Omega}\left(b \widetilde{\Phi}_{t r}\left(\widetilde{\Phi}_{t r}-a\right)\left(\widetilde{\Phi}_{t r}-1\right)+e^{-\varepsilon t} W_{0} \widetilde{\Phi}_{t r}+\varepsilon \kappa e^{-\varepsilon t} \widetilde{\Phi}_{t r} \int_{0}^{t} \widetilde{\Phi}_{t r}(x, \vartheta) e^{\varepsilon \vartheta} d \vartheta\right)^{2} d x \\
& \leqslant C \int_{\Omega}\left(\widetilde{\Phi}_{t r}^{2}\left(\widetilde{\Phi}_{t r}^{2}+a^{2}\right)\left(\widetilde{\Phi}_{t r}^{2}+1\right)+\left|W_{0}\right|^{2} \widetilde{\Phi}_{t r}^{2}+\varepsilon^{2} \kappa^{2} \widetilde{\Phi}_{t r}^{2}\left(\int_{0}^{t}\left|\widetilde{\Phi}_{t r}(x, \vartheta)\right| \cdot\left|e^{\varepsilon \vartheta}\right| d \vartheta\right)^{2}\right) d x \\
& \leqslant C\left(\int_{\Omega}\left|\widetilde{\Phi}_{t r}\right|^{6} d x+\int_{\Omega}\left|\widetilde{\Phi}_{t r}\right|^{4} d x+\int_{\Omega}\left|\widetilde{\Phi}_{t r}\right|^{2} d x\right. \\
& \left.+\int_{\Omega}\left|W_{0} \cdot \widetilde{\Phi}_{t r}\right|^{2} d x+e^{2 \varepsilon T} \int_{\Omega} \widetilde{\Phi}_{t r}^{2} \cdot\left(\int_{0}^{T}\left|\widetilde{\Phi}_{t r}(x, \vartheta)\right| d \vartheta\right)^{2} d x\right) \Longrightarrow \\
& \left\|I_{i o n}\left(\widetilde{\Phi}_{t r}(t), \widetilde{W}(t)\right)\right\|_{L^{2}(\Omega)}^{4 / 3} \leqslant C\left(\int_{\Omega}\left|\widetilde{\Phi}_{t r}\right|^{6} d x+\int_{\Omega}\left|\widetilde{\Phi}_{t r}\right|^{4} d x+\int_{\Omega}\left|\widetilde{\Phi}_{t r}\right|^{2} d x\right. \\
& \left.+\int_{\Omega}\left|W_{0} \cdot \widetilde{\Phi}_{t r}\right|^{2} d x+\int_{\Omega} \widetilde{\Phi}_{t r}^{2} \cdot\left(\int_{0}^{T}\left|\widetilde{\Phi}_{t r}(x, \vartheta)\right| d \vartheta\right)^{2} d x\right)^{2 / 3} \\
& \leqslant C\left(\int_{\Omega}\left|\widetilde{\Phi}_{t r}\right|^{6} d x\right)^{2 / 3}+C\left(1+\int_{\Omega}\left|\widetilde{\Phi}_{t r}\right|^{4} d x+\int_{\Omega}\left|\widetilde{\Phi}_{t r}\right|^{2} d x\right. \\
& \left.+\int_{\Omega}\left|W_{0} \cdot \widetilde{\Phi}_{t r}\right|^{2} d x+\int_{\Omega} \widetilde{\Phi}_{t r}^{2} \cdot\left(\int_{0}^{T}\left|\widetilde{\Phi}_{t r}(x, \vartheta)\right| d \vartheta\right)^{2} d x\right)^{2 / 3} \\
& \leqslant C\left(\int_{\Omega}\left|\widetilde{\Phi}_{t r}\right|^{6} d x\right)^{2 / 3}+C\left(1+\int_{\Omega}\left|\widetilde{\Phi}_{t r}\right|^{4} d x+\int_{\Omega}\left|\widetilde{\Phi}_{t r}\right|^{2} d x\right. \\
& \left.+\int_{\Omega}\left|W_{0} \cdot \widetilde{\Phi}_{t r}\right|^{2} d x+\int_{\Omega} \widetilde{\Phi}_{t r}^{2} \cdot\left(\int_{0}^{T}\left|\widetilde{\Phi}_{t r}(x, \vartheta)\right| d \vartheta\right)^{2} d x\right) \\
& \leqslant C\left(1+\left\|\widetilde{\Phi}_{t r}\right\|_{L^{4}\left[(0, T), L^{6}(\Omega)\right]}^{4}+\left\|\widetilde{\Phi}_{t r}\right\|_{L^{4}\left[(0, T), L^{4}(\Omega)\right]}^{4}+\left\|\widetilde{\Phi}_{t r}\right\|_{L^{2}\left[(0, T), L^{2}(\Omega)\right]}^{2} .\right. \\
& \left.+\left\|\mathrm{W}_{0}\right\|_{L^{\infty}(\Omega)}^{2} \cdot\left\|\widetilde{\Phi}_{t r}\right\|_{L^{2}\left[(0, T), L^{2}(\Omega)\right.}^{2}\right)+C \int_{0}^{T} \int_{\Omega} \widetilde{\Phi}_{t r}^{2}(x, t) \cdot\left(\int_{0}^{T}\left|\widetilde{\Phi}_{t r}(x, \vartheta)\right| d \vartheta\right)^{2} d x d t
\end{aligned}
$$

Due to the continuous imbeddings $L^{4}\left[(0, T), L^{6}(\Omega)\right] \hookrightarrow L^{4}\left(\Omega_{T}\right)=L^{4}\left[\Omega, L^{4}(0, T)\right] \hookrightarrow L^{4}[\Omega$, $\left.L^{2}(0, T)\right],{ }^{30)}$ all norms within the brackets on the right-hand side of (3.41) are finite. The last term can be estimated by

$$
\begin{aligned}
& \int_{0}^{T} \int_{\Omega} \widetilde{\Phi}_{t r}^{2}(x, t) \cdot\left(\int_{0}^{T}\left|\widetilde{\Phi}_{t r}(x, \vartheta)\right| d \vartheta\right)^{2} d x d t=\int_{\Omega} \int_{0}^{T} \widetilde{\Phi}_{t r}^{2}(x, t) d t \cdot\left(\int_{0}^{T}\left|\widetilde{\Phi}_{t r}(x, \vartheta)\right| d \vartheta\right)^{2} d x \\
& \quad=\int_{\Omega}\left\|\widetilde{\Phi}_{t r}(x)\right\|_{L^{2}(0, T)}^{2} \cdot\left\|\widetilde{\Phi}_{t r}(x)\right\|_{L^{1}(0, T)}^{2} d x \leqslant \int_{\Omega}\left\|\widetilde{\Phi}_{t r}(x)\right\|_{L^{2}(0, T)}^{2} \cdot\left\|\widetilde{\Phi}_{t r}(x)\right\|_{L^{2}(0, T)}^{2} d x \\
& \quad \leqslant\left\|\widetilde{\Phi}_{t r}\right\|_{L^{4}\left[\Omega, L^{2}(0, T)\right]}^{4},
\end{aligned}
$$

which is finite as well, and the claim has been proved.

- Step 3. The functions $\widetilde{\Phi}_{t r}=\Phi_{t r}, \widetilde{\Phi}_{e}=\Phi_{e}$ and $\widetilde{W}=W$ belong to the spaces

$$
\begin{aligned}
& \Phi_{t r} \in W^{1,4 / 3}\left[(0, T), L^{2}(\Omega)\right] \cap L^{4 / 3}\left[(0, T), W^{2,2}(\Omega)\right] \cap L^{s}\left(\Omega_{T}\right), 4 \leqslant s<\infty ; \\
& \Phi_{e} \in L^{2}\left[[0, T), L^{2}(\Omega)\right] \cap L^{4 / 3}\left[(0, T), W^{2,2}(\Omega)\right] ; \\
& W \in C^{1}\left[(0, T), L^{\infty}(\Omega)\right] \cap C^{0}\left[[0, T], L^{\infty}(\Omega)\right] .
\end{aligned}
$$

30) For the last imbedding, cf. [Kunisch/Wagner 11] , p. 21, Proposition 4.3. 
In view of Step 2, we may apply Proposition 3.6., Theorem 3.2. and Proposition 3.7. to the triple $\left(\widetilde{\Phi}_{t r}, \widetilde{\Phi}_{e}\right.$, $\widetilde{W})$. Consequently, the according solution $\left(\Phi_{t r}, \Phi_{e}, W\right)$ of $(\widetilde{\mathrm{B}})_{0, g a t}$ and $(\widetilde{\mathrm{B}})_{0, p a r}$ coincides with $\left(\widetilde{\Phi}_{t r}, \widetilde{\Phi}_{e}, \widetilde{W}\right)$ for almost all $t \in(0, T)$ in the first and second component, and for all $t \in[0, T]$ for the third component. This proves the claimed regularity except for the relation $\Phi_{t r} \in L^{s}\left(\Omega_{T}\right)$, which will be derived from the Aubin-Dubinskij-Lions lemma: ${ }^{31)}$ Choosing $\mathrm{X}_{0}=W^{2,2}(\Omega), \mathrm{X}=C^{0}(\Omega)$ and $\mathrm{X}_{1}=L^{2}(\Omega)$, we find that

$$
\Phi_{t r} \in\left\{f \in L^{4 / 3}\left[(0, T), W^{2,2}(\Omega)\right] \mid \frac{d f}{d t} \in L^{4 / 3}\left[(0, T), L^{2}(\Omega)\right]\right\}
$$

belongs to $L^{s}\left[(0, T), C^{0}(\Omega)\right] \subset L^{s}\left(\Omega_{T}\right)$ for all $1<s<\infty$. Hereby the claimed regularity of $W$ with respect to the spatial variables is confirmed as well.

- Step 4. The function $I_{i o n}\left(\widetilde{\Phi}_{t r}, W\right)=I_{i o n}\left(\widetilde{\Phi}_{t r}, \widetilde{W}\right)$ belongs even to the space $L^{6}\left[(0, T), L^{2}(\Omega)\right]$. We estimate

$$
\begin{aligned}
& \left\|I_{i o n}\left(\widetilde{\Phi}_{t r}(t), \widetilde{W}(t)\right)\right\|_{L^{6}(\Omega)}^{6}=\int_{\Omega}\left(I_{i o n}\left(\widetilde{\Phi}_{t r}(t), \widetilde{W}(t)\right)\right)^{6} d x \\
& =\int_{\Omega}\left(b \widetilde{\Phi}_{t r}\left(\widetilde{\Phi}_{t r}-a\right)\left(\widetilde{\Phi}_{t r}-1\right)+e^{-\varepsilon t} W_{0} \widetilde{\Phi}_{t r}+\varepsilon \kappa e^{-\varepsilon t} \widetilde{\Phi}_{t r} \int_{0}^{t} \widetilde{\Phi}_{t r}(x, \vartheta) e^{\varepsilon \vartheta} d \vartheta\right)^{6} d x \\
& \leqslant C \int_{\Omega}\left(\widetilde{\Phi}_{t r}^{6}\left(\widetilde{\Phi}_{t r}^{6}+a^{6}\right)\left(\widetilde{\Phi}_{t r}^{6}+1\right)+\left|W_{0}\right|^{6} \widetilde{\Phi}_{t r}^{6}+\widetilde{\Phi}_{t r}^{6}\left(\int_{0}^{t}\left|\widetilde{\Phi}_{t r}(x, \vartheta)\right| \cdot\left|e^{\varepsilon \vartheta}\right| d \vartheta\right)^{6}\right) d x \\
& \leqslant C\left(\int_{\Omega}\left|\widetilde{\Phi}_{t r}\right|^{18} d x+\int_{\Omega}\left|\widetilde{\Phi}_{t r}\right|^{12} d x+\int_{\Omega}\left|\widetilde{\Phi}_{t r}\right|^{6} d x+\int_{\Omega}\left|W_{0}\right|^{6} \cdot\left|\widetilde{\Phi}_{t r}\right|^{6} d x\right. \\
& \left.+\int_{\Omega}\left|\widetilde{\Phi}_{t r}\right|^{6} \cdot\left(\int_{0}^{t}\left|\widetilde{\Phi}_{t r}(x, \vartheta)\right| \cdot\left|e^{\varepsilon \vartheta}\right| d \vartheta\right)^{6} d x\right) \Longrightarrow \\
& \left.\left\|I_{i o n}\left(\widetilde{\Phi}_{t r}(t), \widetilde{W}(t)\right)\right\|_{L^{6}\left[(0, T), L^{6}(\Omega)\right.}^{6}\right]=\int_{0}^{T}\left\|I_{i o n}\left(\widetilde{\Phi}_{t r}(t), \widetilde{W}(t)\right)\right\|_{L^{6}(\Omega)}^{6} d t \\
& \leqslant C\left(1+\left\|\widetilde{\Phi}_{t r}\right\|_{L^{18}\left(\Omega_{T}\right)}^{18}+\left\|\widetilde{\Phi}_{t r}\right\|_{L^{12}\left(\Omega_{T}\right)}^{12}+\left\|\widetilde{\Phi}_{t r}\right\|_{L^{6}\left(\Omega_{T}\right)}^{6}\right. \\
& \left.+\left\|\mathrm{W}_{0}\right\|_{L^{\infty}(\Omega)}^{6} \cdot\left\|\widetilde{\Phi}_{t r}\right\|_{L^{6}\left(\Omega_{T}\right)}^{6}\right)+C \int_{0}^{T} \int_{\Omega}\left|\widetilde{\Phi}_{t r}(x, t)\right|^{6} \cdot\left(\int_{0}^{T}\left|\widetilde{\Phi}_{t r}(x, \vartheta)\right| d \vartheta\right)^{6} d x d t
\end{aligned}
$$

From Step 3 we know that all norms within the brackets on the right-hand side of (3.53) are finite. Analogously to $(3.42)-(3.44)$, the last term will be estimated through

$$
\begin{aligned}
\int_{0}^{T} & \int_{\Omega}\left|\widetilde{\Phi}_{t r}(x, t)\right|^{6} \cdot\left(\int_{0}^{T}\left|\widetilde{\Phi}_{t r}(x, \vartheta)\right| d \vartheta\right)^{6} d x d t=\int_{\Omega} \int_{0}^{T}\left|\widetilde{\Phi}_{t r}(x, t)\right|^{6} d t \cdot\left(\int_{0}^{T}\left|\widetilde{\Phi}_{t r}(x, \vartheta)\right| d \vartheta\right)^{6} d x \\
& =\int_{\Omega}\left\|\widetilde{\Phi}_{t r}(x)\right\|_{L^{6}(0, T)}^{6} \cdot\left\|\widetilde{\Phi}_{t r}(x)\right\|_{L^{1}(0, T)}^{6} d x \leqslant C \int_{\Omega}\left\|\widetilde{\Phi}_{t r}(x)\right\|_{L^{6}(0, T)}^{6} \cdot\left\|\widetilde{\Phi}_{t r}(x)\right\|_{L^{6}(0, T)}^{6} d x \\
& =\left\|\widetilde{\Phi}_{t r}\right\|_{L^{12}\left[\Omega, L^{6}(0, T)\right]}^{12}
\end{aligned}
$$

which is by (3.45) finite as well. Now the claim follows from the continuous imbedding $L^{6}\left[(0, T), L^{2}(\Omega)\right] \hookrightarrow$ $L^{6}\left[(0, T), L^{6}(\Omega)\right]$.

- Step 5. The functions $\widetilde{\Phi}_{t r}=\Phi_{t r}, \widetilde{\Phi}_{e}=\Phi_{e}$ and $\widetilde{W}=W$ belong even to the spaces

$$
\begin{aligned}
& \Phi_{t r} \in W^{1,6}\left[(0, T), L^{2}(\Omega)\right] \cap L^{6}\left[(0, T), W^{2,2}(\Omega)\right] \cap C^{0}\left(\Omega_{T}\right) ; \\
& \Phi_{e} \in L^{2}\left[[0, T), L^{2}(\Omega)\right] \cap L^{r}\left[(0, T), W^{2,2}(\Omega)\right] ; \\
& W \in C^{1}\left[(0, T), L^{\infty}(\Omega)\right] \cap C^{0}\left[[0, T], L^{\infty}(\Omega)\right] .
\end{aligned}
$$

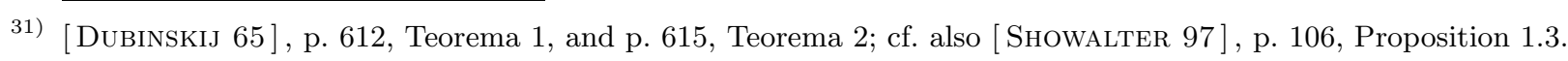


In view of Step 4, Proposition 3.6., Theorem 3.2. and Proposition 3.7. may be applied again to the triple $\left(\widetilde{\Phi}_{t r}, \widetilde{\Phi}_{e}, \widetilde{W}\right)$, and the new solution $\left(\Phi_{t r}, \Phi_{e}, W\right)$ of $(\widetilde{\mathrm{B}})_{0, g a t}$ and $(\widetilde{\mathrm{B}})_{0, p a r}$ coincides again with the former one. Using interpolation space arguments, the claim $\Phi_{t r} \in C^{0}\left(\Omega_{T}\right)$ follows from

$$
\Phi_{t r} \in\left\{f \in L^{6}\left[(0, T), W^{2,2}(\Omega)\right] \mid \frac{d f}{d t} \in L^{6}\left[(0, T), L^{2}(\Omega)\right]\right\}
$$

by repeating the arguments from [VENERONI 09], p. $864 \mathrm{f}$.

- Step 6. Conclusion of the proof. Let us finally note that the regularity $(3.14)-(3.16)$ of a weak solution $\left(\Phi_{t r}, \Phi_{e}, W\right)$ of $(\mathrm{B})_{2}$ allows for partial integration in all terms where $\nabla \psi$ occurs. At the same time, it is consistent with Definition 2.1. Consequently, $\left(\Phi_{t r}, \Phi_{e}, W\right)$ forms a strong solution of $(\mathrm{B})_{1}$ and $(\mathrm{B})_{0}$ as well, and the proof of Theorem 3.3. is complete.

Remarks. 1) If the Rogers-McCulloch model in $(\mathrm{B})_{2}$ is replaced by the FitzHugh-Nagumo model then the proof of Theorem 3.3. runs as above with only minor alterations in Steps 2 and 4.

2) In the case of the linearized Aliev-Panfilov model, the relation $\Phi_{t r} \in L^{4}\left[(0, T), L^{6}(\Omega)\right]$ is confirmed as above. However, Step 2 of the preceding proof must be replaced by the following calculations, which show again that the function $I_{i o n}\left(\widetilde{\Phi}_{t r}, W\right)=I_{i o n}\left(\widetilde{\Phi}_{t r}, \widetilde{W}\right)$ belongs to the space $L^{4 / 3}\left[(0, T), L^{2}(\Omega)\right]$ :

$$
\begin{aligned}
& \left\|I_{i o n}\left(\widetilde{\Phi}_{t r}(t), \widetilde{W}(t)\right)\right\|_{L^{2}(\Omega)}^{2}=\int_{\Omega}\left(I_{i o n}\left(\widetilde{\Phi}_{t r}(t), \widetilde{W}(t)\right)\right)^{2} d x \\
& =\int_{\Omega}\left(b \widetilde{\Phi}_{t r}\left(\widetilde{\Phi}_{t r}-a\right)\left(\widetilde{\Phi}_{t r}-1\right)+e^{-\varepsilon t} W_{0} \widetilde{\Phi}_{t r}+\varepsilon \kappa e^{-\varepsilon t} \widetilde{\Phi}_{t r} \int_{0}^{t}\left((a+1) \widetilde{\Phi}_{t r}(x, \vartheta)-\widetilde{\Phi}_{t r}^{2}(x, \vartheta)\right) e^{\varepsilon \vartheta} d \vartheta\right)^{2} d x \\
& \leqslant C \int_{\Omega}\left(\widetilde{\Phi}_{t r}^{2}\left(\widetilde{\Phi}_{t r}^{2}+a^{2}\right)\left(\widetilde{\Phi}_{t r}^{2}+1\right)+\left|W_{0}\right|^{2} \widetilde{\Phi}_{t r}^{2}\right. \\
& \left.+\varepsilon^{2} \kappa^{2} \widetilde{\Phi}_{t r}^{2}\left(\int_{0}^{t}\left(\left|(a+1) \widetilde{\Phi}_{t r}(x, \vartheta)\right|+\left|\widetilde{\Phi}_{t r}(x, \vartheta)\right|^{2}\right) \cdot\left|e^{\varepsilon \vartheta}\right| d \vartheta\right)^{2}\right) d x \\
& \leqslant C\left(\int_{\Omega}\left|\widetilde{\Phi}_{t r}\right|^{6} d x+\int_{\Omega}\left|\widetilde{\Phi}_{t r}\right|^{4} d x+\int_{\Omega}\left|\widetilde{\Phi}_{t r}\right|^{2} d x+\int_{\Omega}\left|W_{0} \cdot \widetilde{\Phi}_{t r}\right|^{2} d x\right. \\
& \left.+e^{2 \varepsilon T} \int_{\Omega} \widetilde{\Phi}_{t r}^{2} \cdot\left(\int_{0}^{T}\left(\left|(a+1) \widetilde{\Phi}_{t r}(x, \vartheta)\right|+\left|\widetilde{\Phi}_{t r}(x, \vartheta)\right|^{2}\right) d \vartheta\right)^{2} d x\right) \Longrightarrow \\
& \left\|I_{\text {ion }}\left(\widetilde{\Phi}_{t r}(t), \widetilde{W}(t)\right)\right\|_{L^{2}(\Omega)}^{4 / 3} \leqslant C\left(\int_{\Omega}\left|\widetilde{\Phi}_{t r}\right|^{6} d x+\int_{\Omega}\left|\widetilde{\Phi}_{t r}\right|^{4} d x+\int_{\Omega}\left|\widetilde{\Phi}_{t r}\right|^{2} d x\right. \\
& \left.+\int_{\Omega}\left|W_{0} \cdot \widetilde{\Phi}_{t r}\right|^{2} d x+\int_{\Omega} \widetilde{\Phi}_{t r}^{2} \cdot\left(\int_{0}^{T}\left(\left|(a+1) \widetilde{\Phi}_{t r}(x, \vartheta)\right|+\left|\widetilde{\Phi}_{t r}(x, \vartheta)\right|^{2}\right) d \vartheta\right)^{2} d x\right)^{2 / 3} \\
& \leqslant C\left(\int_{\Omega}\left|\widetilde{\Phi}_{t r}\right|^{6} d x\right)^{2 / 3}+C\left(1+\int_{\Omega}\left|\widetilde{\Phi}_{t r}\right|^{4} d x+\int_{\Omega}\left|\widetilde{\Phi}_{t r}\right|^{2} d x\right. \\
& \left.+\int_{\Omega}\left|W_{0} \cdot \widetilde{\Phi}_{t r}\right|^{2} d x+\int_{\Omega} \widetilde{\Phi}_{t r}^{2} \cdot\left(\int_{0}^{T}\left(\left|(a+1) \widetilde{\Phi}_{t r}(x, \vartheta)\right|+\left|\widetilde{\Phi}_{t r}(x, \vartheta)\right|^{2}\right) d \vartheta\right)^{2} d x\right)^{2 / 3} \\
& \leqslant C\left(\int_{\Omega}\left|\widetilde{\Phi}_{t r}\right|^{6} d x\right)^{2 / 3}+C\left(1+\int_{\Omega}\left|\widetilde{\Phi}_{t r}\right|^{4} d x+\int_{\Omega}\left|\widetilde{\Phi}_{t r}\right|^{2} d x\right. \\
& \left.+\int_{\Omega}\left|W_{0} \cdot \widetilde{\Phi}_{t r}\right|^{2} d x+\int_{\Omega} \widetilde{\Phi}_{t r}^{2} \cdot\left(\int_{0}^{T}\left(\left|(a+1) \widetilde{\Phi}_{t r}(x, \vartheta)\right|+\left|\widetilde{\Phi}_{t r}(x, \vartheta)\right|^{2}\right) d \vartheta\right)^{2} d x\right) \Longrightarrow \\
& \left\|I_{i o n}\left(\widetilde{\Phi}_{t r}, \widetilde{W}\right)\right\|_{L^{4 / 3}\left[(0, T), L^{2}(\Omega)\right.}^{4 / 3}=\int_{0}^{T}\left\|I_{i o n}\left(\widetilde{\Phi}_{t r}(t), \widetilde{W}(t)\right)\right\|_{L^{2}(\Omega)}^{4 / 3} d t \\
& \leqslant C\left(1+\left\|\widetilde{\Phi}_{t r}\right\|_{L^{4}\left[(0, T), L^{6}(\Omega)\right.}^{4}+\left\|\widetilde{\Phi}_{t r}\right\|_{L^{4}\left[(0, T), L^{4}(\Omega)\right.}^{4}+\left\|\widetilde{\Phi}_{t r}\right\|_{L^{2}\left[(0, T), L^{2}(\Omega)\right.}^{2}\right] \\
& \left.\left.+\left\|\mathrm{W}_{0}\right\|_{L^{\infty}(\Omega)}^{2} \cdot\left\|\widetilde{\Phi}_{t r}\right\|_{L^{2}\left[(0, T), L^{2}(\Omega)\right]}^{2}\right]\right)
\end{aligned}
$$




$$
+C \int_{0}^{T} \int_{\Omega} \widetilde{\Phi}_{t r}^{2}(x, t) \cdot\left(\int_{0}^{T}\left(\left|(a+1) \widetilde{\Phi}_{t r}(x, \vartheta)\right|+\left|\widetilde{\Phi}_{t r}(x, \vartheta)\right|^{2}\right) d \vartheta\right)^{2} d x d t .
$$

The finiteness of the norms within the brackets on the right-hand side of (3.67) is confirmed as in the proof above. For the last term, we get the estimate

$$
\begin{aligned}
& \int_{0}^{T} \int_{\Omega} \widetilde{\Phi}_{t r}^{2}(x, t) \cdot\left(\int_{0}^{T}\left(\left|(a+1) \widetilde{\Phi}_{t r}(x, \vartheta)\right|+\left|\widetilde{\Phi}_{t r}(x, \vartheta)\right|^{2}\right) d \vartheta\right)^{2} d x d t \\
& =\int_{\Omega} \int_{0}^{T} \widetilde{\Phi}_{t r}^{2}(x, t) d t \cdot\left(\int_{0}^{T}\left|(a+1) \widetilde{\Phi}_{t r}(x, \vartheta)\right| d \vartheta+\int_{0}^{T} \widetilde{\Phi}_{t r}^{2}(x, \vartheta) d \vartheta\right)^{2} d x \\
& \leqslant \int_{\Omega}\left\|\widetilde{\Phi}_{t r}(x)\right\|_{L^{2}(0, T)}^{2} \cdot\left(\left\|\widetilde{\Phi}_{t r}(x)\right\|_{L^{1}(0, T)}^{2}+\left\|\widetilde{\Phi}_{t r}(x)\right\|_{L^{2}(0, T)}^{4}\right) d x \\
& \leqslant C \int_{\Omega}\left\|\widetilde{\Phi}_{t r}(x)\right\|_{L^{2}(0, T)}^{2} \cdot\left(\left\|\widetilde{\Phi}_{t r}(x)\right\|_{L^{2}(0, T)}^{2}+\left\|\widetilde{\Phi}_{t r}(x)\right\|_{L^{2}(0, T)}^{4}\right) d x \\
& \leqslant C\left(\left\|\widetilde{\Phi}_{t r}\right\|_{L^{4}\left[\Omega, L^{2}(0, T)\right]}^{4}+\left\|\widetilde{\Phi}_{t r}\right\|_{L^{6}\left[\Omega, L^{2}(0, T)\right]}^{6}\right) .
\end{aligned}
$$

As in Step 2 in the proof above, we see that the first member on the right-hand side is finite. Due to [Kunisch/Wagner 11] , p. 21, Proposition 4.3., the imbeddings $L^{4}\left[(0, T), L^{6}(\Omega)\right] \hookrightarrow L^{6}\left[\Omega, L^{4}(0, T)\right]$ $\hookrightarrow L^{6}\left[\Omega, L^{2}(0, T)\right]$ are continuous, and the second member is finite as well. Step 4 is subjected to analogous alterations.

Proof of Corollary 3.4. Under the assumptions of the Corollary, $(\mathrm{B})_{2}$ possesses a uniquely determined weak solution $\left(\Phi_{t r}, \Phi_{e}, W\right)$ on $[0, T]$ by Theorem 2.8. By Theorem 3.3., this solution admits the regularity described in $(3.14)-(3.16)$ and is, in fact, a strong solution of $(B)_{0}$ and $(B)_{1}$.

Proof of Corollary 3.5. 1) By Theorem 3.3., any weak solution of $(B)_{2}$ is in fact a strong solution of $(\mathrm{B})_{0}$ and $(\mathrm{B})_{1}$ with $\Phi_{t r}, \Phi_{e} \in L^{r}\left[(0, T), W^{2,2}(\Omega)\right]$ at least. By Assumption 3.1., 1), the boundary of $\Omega$ is $C^{1,1}$, and by Assumption 3.1., 2), the entries of $M_{i}$ and $M_{e}$ are Lipschitz continuous. Since the eigenvalues $\lambda_{k, i}(\cdot)$ and $\lambda_{k, e}(\cdot), 1 \leqslant k \leqslant 3$, depend Lipschitz-continuously on the matrix elements (cf. [SCHABACK/WEndland 05] , p. 270, Corollary 15.4.), the functions are continuous on $\mathrm{cl}(\Omega)$. Now the assertion follows from [Bourgault/Coudiére/Pierre 09], p. 462, Lemma 1.

2) Under the assumptions above the compatibility condition for $\Phi_{0}$ reduces to $\mathfrak{n}^{\mathrm{T}} M_{e} \nabla \Phi_{0}=0$ on $\partial \Omega$ after [Veneroni 09] , p. 854, Remark 3. By Part 1, this condition may be further reduced to the claimed form.

\section{Acknowledgement.}

The present work has been supported within the Special Research Unit "Mathematical Optimization and Applications in Biomedical Sciences" (Graz) by the Austrian Science Fund.

\section{References.}

1. [Adams/Fournier 07] Adams, R. A.; Fournier, J. J. F.: Sobolev Spaces. Academic Press / Elsevier; Amsterdam etc. 2007, 2nd ed.

2. [Aliev/Panfilov 96] Aliev, R. R.; Panfilov, A. V.: A simple two-variable model of cardiac excitation. Chaos, Solitons \& Fractals 7 (1996), $293-301$

3. [Boulakia/Fernández/Gerbeau/Zemzemi 08] Boulakia, M.; Fernández, M. A.; Gerbeau, J.-F.; Zemzemi, N.: A coupled system of PDEs and ODEs arising in electrocardiograms modeling. Appl. Math. Res. Express (2008), http://dx.doi.org/10.1093/amrx/abn002 (electronically published) 
4. [Bourgault/Coudière/Pierre 09] Bourgault, Y.; Coudière, Y.; Pierre, C.: Existence and uniqueness of the solution for the bidomain model used in cardiac electrophysiology. Nonlinear Analysis: Real World Appl. 10 (2009), $458-482$

5. [ Colli Franzone/Savaré 02] Colli Franzone, P.; Savaré, G.: Degenerate evolution systems modeling the cardiac electric field at micro- and macroscopic level. In: Lorenzi, A.; Ruf, B. (Eds.): Evolution Equations, Semigroups and Functional Analysis. Birkhäuser; Basel - Boston - Berlin 2002 (Progress in Nonlinear Differential Equations and their Applications, Vol. 50), $49-78$

6. [Colli Franzone/Guerri/Tentoni 90] Colli Franzone, P.; Guerri, L.; Tentoni, S.: Mathematical modeling of the excitation process in myocardial tissue: influence of fiber rotation on wavefront propagation and potential field. Math. Biosciences 101 (1990), 155 - 235

7. [Dubinskij 65] Dubinskij, Ju. А. (Дубинский, Ю. А.): Слабая сходимость в нелинейных әллиптических и параболических уравнениях. Мат. Сборник 67 (1965) 4, 609 - 642

8. [Evans 98] Evans, L. C.: Partial Differential Equations. American Mathematical Society; Providence 1998

9. [FitzHugh 61] FitzHugh, R.: Impulses and physiological states in theoretical models of nerve membrane. Biophysical J. 1 (1961), $445-466$

10. [ Ітто/Kunisch 08] Ito, K.; Kunisch, K.: Lagrange Multiplier Approach to Variational Problems and Applications. SIAM; Philadelphia 2008

11. [Kunisch/Wagner 11] Kunisch, K.; Wagner, M.: Optimal control of the bidomain system (I): The monodomain approximation with the Rogers-McCulloch model. Revised version. University of Leipzig, Institute of Mathematics, Preprint No. 03/2011

12. [Nagaiah/Kunisch/Plank 11] Nagaiah, C.; Kunisch, K.; Plank, G.: Numerical solution for optimal control of the reaction-diffusion equations in cardiac electrophysiology. Comput. Optim. Appl. 49 (2011), 149 - 178

13. [Nagumo/Arimoto/Yoshizawa 62] Nagumo, J.; Arimoto, S.; Yoshizawa, S.: An active pulse transmission line simulating nerve axon. Proceedings of the Institute of Radio Engineers 50 (1962), 2061 - 2070

14. [Rogers/McCulloch 94] Rogers, J. M.; McCulloch, A. D.: A collocation-Galerkin finite element model of cardiac action potential propagation. IEEE Transactions of Biomedical Engineering 41 (1994), 743 - 757

15. [Schaback/Wendland 05] Numerische Mathematik. Springer; Berlin etc. 2005. 5. ed.

16. [Showalter 97] Showalter, R. E.: Monotone Operators in Banach Space and Nonlinear Partial Differential Equations. American Mathematical Society; Providence 1997

17. [Sundnes/Lines/Cai/Nielsen/Mardal/Tveito 06] Sundnes, J.; Lines, G. T.; Cai, X.; Nielsen, B. F.; Mardal, K.-A.; Tveito, A.: Computing the Electrical Activity in the Heart. Springer; Berlin etc. 2006

18. [Tung 78] Tung, L.: A Bi-Domain Model for Describing Ischemic Myocardial D-C Potentials. PhD thesis. Massachusetts Institute of Technology 1978

19. [Veneroni 09] Veneroni, M.: Reaction-diffusion systems for the macroscopic bidomain model of the cardiac electric field. Nonlinear Analysis: Real World Applications 10 (2009), 849 - 868

20. [WArga 72] Warga, J.: Optimal Control of Differential and Functional Equations. Academic Press; New York London 1972

Last modification: 20.10. 2011

Authors' addresses / e-mail. Karl Kunisch: University of Graz, Institute for Mathematics and Scientific Computing, Heinrichstraße 36, A-8010 Graz, Austria. e-mail: karl.kunisch @ uni-graz.at

Marcus Wagner: University of Leipzig, Department of Mathematics, P. O. B. 1009 20, D-04009 Leipzig, Germany. Homepage / e-mail: www.thecitytocome.de / marcus . wagner @ math. uni-leipzig.de 